\title{
Dynamic Modelling and Operational Optimisation of Natural Draft Cooling Towers
}

\author{
A. Dhorat ${ }^{1}$, M. A. Al-Obaidi ${ }^{1,2}$ and I.M. Mujtaba ${ }^{1, *}$ \\ ${ }^{1}$ Chemical Engineering Division, School of Engineering, University of Bradford. \\ Bradford, West Yorkshire BD7 1DP, UK \\ ${ }^{2}$ Middle Technical University, Iraq - Baghdad \\ *Corresponding author, Tel.: +44 01274233645 \\ E-mail address: $\underline{\text { I.M.Mujtaba@ bradford.ac.uk }}$
}

\begin{abstract}
Natural draft cooling towers are subjected to dynamic air temperature and humidity ratio of air throughout the day. Their performances based on steady state model is restricted to single air temperature and humidity ratio of air values and thus are not accurate. In this work, a dynamic model for a natural draft cooling tower is developed. Air temperature and relative humidity data for Johannesburg were acquired to develop dynamic correlations for these two parameters and are embedded in the process model together with the dynamic model of the collection basin of the tower. A simple algorithm is proposed to solve the resulting boundary value problem. Finally, the common perception that seawater/river water should be used instead of freshwater as cooling medium is tested via optimisation where the operating costs of the process for two cooling mediums is minimised. The freshwater has been found to be cost effective cooling medium.
\end{abstract}

Keywords: Cooling Towers; Numerical Modelling; Dynamic Modelling; SQP Optimisation; Cycles of Concentration

\section{Introduction}

Cooling processes such as wet-cooling towers are commonly used to reject waste heat which are associated with several industrial processes such as power generation, chemical and process plants. Precisely, a cooling tower is a unit operation, which completes a heat exchanger network (HEN) so that cooling water can be recirculated around a system. The mechanism of a wet natural draft cooling tower works by the principle of evaporative heat transfer where an air stream is in contact with a water stream (Williamson et al., 2008). Figure 1 shows a schematic of a natural draft cooling tower. It can be seen that the air and water stream are in contact and the humidity of the air would increase as the air is drawn up the tower. The main driving force is down to mass transfer whereby the water at the interface 
would begin to evaporate, as there is enough energy produced for the water to change state by obtaining the latent heat of vaporisation.

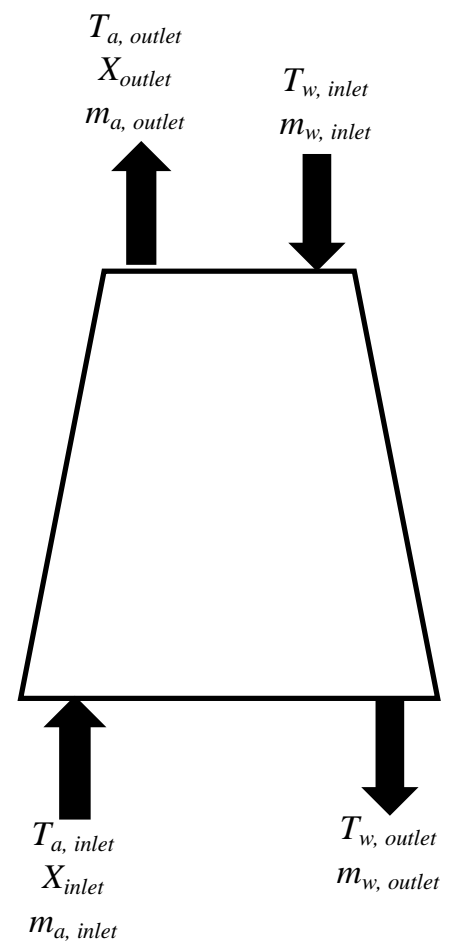

Figure 1. Schematic diagram of a natural draft cooling tower

As the heat ejection is dependent on evaporative heat transfer, the variable air temperature and humidity ratios a tower is exposed to can have a significant impact on the performance and operating costs.

Al-Nimr (1998) presented an analytical dynamic model for a cooling tower, which accounted for time and axial distributions. However, the key assumptions used in his work was that the mass and air flowrates into the tower were uniform and the unsteadiness in the thermal performance had arisen from the step changes in the inlet temperatures of both streams. Conversely, this model only presented an approximate closed form solution as the exact solution was only presented for a steady state model.

Kloppers and Kroger (2004) utilised a leapfrog optimisation program with constraints (LFOPC) optimisation algorithm, which is different to traditional sequential quadratic 
programming methods (SQP), as it does not use any line searches. The objective functions in their work explored the total cost, which is the sum of the total operating cost and capital costs. Results had shown good outputs, which were feasible from an engineering perspective. However, the limitations of this work include the fact that this analysis was based upon steady state modelling, which did not account for the variation in air temperature throughout the period of a year.

Kloppers and Kroger (2005) investigated the influence of the Lewis factor on the prediction of natural draft cooling towers in the unsaturated state. Results show that at high ambient air temperatures and in very humid conditions, the Lewis factor has very little influence on the heat rejection rates. However, at lower temperatures and low humidities, the effect of the Lewis factor becomes more pronounced as heat rejection rates can vary as much as $5 \%$ when utilising a Lewis factor of 0.5 or 1.3. As the Lewis factor was not modelled in the supersaturated state, the influence of the Lewis factor would be more pronounced in large cooling towers as it would be dictated by the humidity differential between the saturation humidity evaluated at water temperature and the saturation humidity evaluated at the air temperature. As the difference between the two saturation humidities converge in the supersaturated state, the Lewis factor would reduce along the height of the cooling tower due to the warming of the water stream through giving up the latent heat of vaporisation and condensation occurring.

Cortinovis et al. (2009) developed an integrated model of a cooling tower and a network of heat exchangers based on a pilot scale network. In his study, they set the objective to optimise the fan speed, water removal flow rate and valve positions at the heat exchanger branches. Results showed that the optimal operational parameters included keeping the water inlet temperature as high as possible. However, the validity of it must be questioned when optimising a full-scale tower as the developed model was only applied to pilot scale.

Ortega et al. (2010) demonstrated a full superstructure approach towards the synthesis of a cooler network. The model was based on disjunctive programming formulation, which ended up as an MINLP problem. The main objective was to minimise the total annual cost and three example problems were given to show the application of the approach. Results showed similar outputs to Cortinovis et al. (2009) with respect to the optimised case, the maximum inlet temperature of the cooling tower was critical for the optimised design. The model was based on a single equation, which means that the accuracy of the output data is questionable without further validation. 
Gololo and Majozi (2011) presented a technique for the grassroots design for a cooling water system in order to minimise wastewater. A heat exchanger network was synthesised with multiple cooling towers based on a superstructure approach. An NLP and MINLP formulation approach was undertaken. Results had shown that the operating capacity of the network was debottlenecked without compromising heat duties.

Klimanek (2013) developed a steady state one-dimensional model of a cooling tower. The model was validated against existing plant data and the system of equations had yielded results, which allowed the temperature and humidity ratio distributions to be seen along the height of the tower. As the equations are only ordinary differential equations, they can be added to a system of partial differential equations in order to develop it into a dynamic model.

He et al. (2016) outlined three mathematical models and iterative algorithms for a natural draft dry cooling tower, a pre-cooled natural draft dry cooling tower and a natural draft wet cooling tower. The Merkel analysis was employed with respect to the natural draft wet cooling tower model. However, the limitations of the Merkel method include the fact that it does not account for saturation within the tower, rather it is the air leaving the tower, which is assumed to be saturated. Furthermore, the water lost through evaporation is not accounted for within the energy balance. The Lewis factor is also assumed to be unity as the tower is assumed to be in an unsaturated state. The specific heats of the air-water mixture were also assumed to be constant throughout the tower. As they modelled the system over the period of a year, questions must be asked during the spring months. As the humidity increases, the likelihood of supersaturation occurring within the tower would be high. As the evaporation rate was assumed to be negligible within the energy balance, the results outlined throughout the year does not correspond to the physics of the natural draft cooling tower. For example, in the months of August to October, the temperature increases from $14{ }^{\circ} \mathrm{C}$ to $27.4{ }^{\circ} \mathrm{C}$. However, the relative humidity has a sharp decrease from $36.9 \%$ to $16.8 \%$ along with the mass flowrate of air decreasing by $33 \%$. The results had shown that the evaporation rate had remained constant, which would show that the change in the temperature, air mass flowrate and humidity had an equal effect. Note, the assumptions used in this work may lead to slight inaccuracies in the results.

As the discussions outlined in this section has focused on the different types of modelling of cooling towers in literature, it has been observed that with the exception of experiments, to the author's knowledge, no literature paper has solved a rigorous dynamic model of a cooling tower with plant data which accounts for saturation and supersaturation. Also, the type of 
cooling medium can have significant ramifications for the design and operation of a cooling tower. When freshwater is used as the cooling medium, the risk of fouling is expected to be much lower than using river water or seawater as the cooling medium. This is down to the fact that there are less total dissolved solids (TDS) within freshwater and therefore the likelihood of solid deposition in the fill and in the collection basin is to be lower (Alsadaie and Mujtaba, 2017). Further considerations include the fact that the operating cost when using freshwater would be lower due to less chemical treatment costs affiliated with antiscalants and blowdown. However, the common perception is that as freshwater scarcity is becoming an ever-increasing problem, the use of river water or seawater as a cooling medium must be considered as a sustainable alternative.

Therefore, the aims of this paper are to:

(a) develop dynamic correlations for temperature of air and the humidity ratio using the data of an existing cooling tower in Johannesburg. Thereafter, the correlations will be coupled with the steady state model of the cooling tower to simulate the dynamic behaviour of the cooling tower. As the evaporation rate is transient, the collection basin at the bottom of the cooling tower will be modelled (dynamic) from first principles and will be incorporated in the above dynamic model before using the model for optimising the process parameters.

(b) evaluate the cost effectiveness of two cooling mediums (i) freshwater (ii) seawater. For each cooling medium three case studies will be investigated. The objective function of each case study will be the hourly operating cost, mass of accumulated water within the basin and the mass flowrate of makeup water. The only constraint used within this optimisation study is the height of the collection basin as optimisation could also be used as a method of control. The parameters, which will be optimised to satisfy the objective function, are the makeup water and number of cycles of concentration.

In comparison to previous literature detailing dynamic models or optimisations, the innovations of this work are many. The evaporation rate is not assumed to be negligible. The change in the mass flowrate of water is modelled in both the unsaturated and supersaturated phases. In the unsaturated phase, the rate of the water mass flowrate being transferred from the interface of water is proportional to the concentration difference between the air-water interface and the bulk air stream. In the supersaturated phase, the water further evaporates due to the main driving force being the difference in the saturation humidity ratio at the water temperature and the saturation humidity ratio at the air temperature. Until the driving force 
(saturation humidities difference) becomes positive, the cooling water temperature will start to cool as it will begin to condense. Therefore, the net evaporation rate presented in this paper accounts for evaporated water and condensed water. The Lewis factors are evaluated at each integration step and are not found to be constant or at unity. Different correlations for the unsaturated state and the supersaturated state are utilised in this model to accurately predict the Lewis factor. Furthermore, the model can output a distribution of temperatures along the height of the tower but this has not been included in this paper as it is beyond the scope of the paper. It would be possible to display the temperature at any point within the tower over a 24hour period as this model uses the height of the tower as a differential variable whereby gPROMs would solve using an adaptive step size routine checking each step for a saturated state or supersaturated state. Finally, the temperatures predicted would be more accurate in comparison to previous works as the specific heats of the water, air and water-air interface were calculated at each integration step along the height of the tower. Beyond the algorithm, the operational cost difference between seawater and freshwater were investigated and presented as well as optimised.

\section{Dynamic Modelling}

\subsection{Cooling Tower Equations (Steady State)}

The governing equations used within this simulation were proposed by Klimanek (2013). For the unsaturated case where the humidity ratio is smaller than the saturation humidity ratio, Eqs. (1) - (5) apply. In the unsaturated state, the mass transfer is characterised by the humidity differential as shown in Eq. (1) and (2). Eqs. (3) and (4) show that the temperature distributions as functions of heat and mass transfer.

$$
\begin{aligned}
& \frac{\partial m_{w}}{\partial z}=\beta a A_{z}\left(X_{s}^{w}-X\right) \\
& \frac{\partial X}{\partial z}=\frac{\beta a A_{z}\left(X_{s}^{w}-X\right)}{m_{a}} \\
& \frac{\partial T_{a}}{\partial z}=\beta a A_{z} \frac{\left[L e_{f}\left(T_{w}-T_{a}\right)\left(C_{p a}^{a}+C_{p v}^{a} X\right)+\left(C_{p v}^{w} T_{w}-C_{p v}^{a} T_{a}\right)\left(X_{s}^{w}-X\right)\right]}{m_{a}\left(C_{p a}^{a}+C_{p v}^{a} X\right)} \\
& \frac{\partial T_{w}}{\partial z}=\beta a A_{z} \frac{\left[L e_{f}\left(T_{w}-T_{a}\right)\left(C_{p a}^{a}+C_{p v}^{a} X\right)+\left(r_{0}+C_{p v}^{w} T_{w}-C_{p w}^{w} T_{w}\right)\left(X_{s}^{w}-X\right)\right]}{m_{w} C_{p w}^{w}} \\
& L e_{f}=0.866^{\frac{2}{3}} \times \frac{\left(\frac{X_{S}^{w}+0.622}{X+0.622}-1\right)}{\ln \left(\frac{X_{S}^{w}+0.622}{X+0.622}\right)}
\end{aligned}
$$


For the supersaturated state where the humidity ratio is larger than the saturation humidity at air temperature, Eqs. (6) - (10) apply. Eqs. (8) and (9) show that the mass transfer is now occurring in the opposite direction whereby water is now condensing instead of evaporating as the saturation humidity at water temperature is lower than the saturation humidity of air. With respect to the temperature distributions, the air temperature will now decrease due to the fact that as water vapour condenses, the latent heat of vaporisation is released and thus would warm the air.

$$
\begin{aligned}
& \frac{\partial m_{w}}{\partial z}=\beta a A_{z}\left(X_{S}^{w}-X_{S}^{a}\right) \\
& \frac{\partial X}{\partial z}=\frac{\beta a A_{z}\left(X_{S}^{w}-X_{S}^{a}\right)}{m_{a}} \\
& \frac{\partial T_{a}}{\partial z}= \\
& -\beta a A_{z} \frac{\left[C_{p a}^{a} L e_{f}\left(T_{a}-T_{w}\right)-X_{s}^{w}\left(r_{0}+C_{p v}^{w} T_{w}\right)+C_{p w}^{a}\left(L e_{f}\left(T_{a}-T_{w}\right)\left(X-X_{S}^{a}\right)+T_{a}\left(X_{s}^{w}-X_{S}^{a}\right)\right)+X_{S}^{a}\left(r_{0}+C_{p v}^{a} T_{w}\right) L e_{f}\left(T_{a}-T_{w}\right)+C_{p v}^{w} T_{w}\right]}{\left[C_{p a}^{a}+C_{p w}^{a} X+\frac{d X_{s}^{a}}{d T_{a}}\left(r_{0}+C_{p v}^{a} T_{a}-C_{p w}^{a} T_{a}\right)+X_{s}^{a}\left(C_{p v}^{a}-C_{p w}^{a}\right)\right]}
\end{aligned}
$$

$\frac{\partial T_{w}}{\partial z}=\beta a A_{z} \frac{\left[\left(r_{0}+C_{p v}^{w} T_{w}-C_{p w}^{w} T_{w}\right)\left(X_{s}^{w}-X_{s}^{a}\right)+L e_{f}\left(T_{w}-T_{a}\right)\left(C_{p a}^{a}+C_{p w}^{a}\left(X-X_{s}^{a}\right)+C_{p v}^{a} X_{S}^{a}\right]\right.}{m_{w} C_{p w}^{w}}$

$L e_{f}=0.866^{\frac{2}{3}} \times \frac{\left(\frac{X_{S}^{w}+0.622}{X_{S}^{a}+0.622}-1\right)}{\ln \left(\frac{X_{S}^{w}+0.622}{X_{S}^{a}+0.622}\right)}$

\subsection{Dynamic Correlations}

It can be seen in Section 2.1. that the model proposed are only in the form of ordinary differential equations as the derivatives are only with respect to the height of the cooling tower and represent a steady state distributed model of the cooling tower. To extend the steady state model into a dynamic model, further correlations are required, which describes the air temperature and humidity ratio of the air changing with time. Therefore, air temperature and relative humidity data over a 24-hour period in Johannesburg was acquired from open literature (AccuWeather, 2017). This 24-hour period was selected due to the fact that there was a large step change in the humidity ratio of the air. As Johannesburg is not near the coast, a day where the humidity ratio changing dramatically is not usually seen. Therefore, for this model which incorporates time; it would be a good test of the robustness of the model when there is a large step change in the humidity ratio and to see its impact on the cooling tower. A psychrometric chart was used to determine the humidity ratio at each temperature interval. Time dependent correlations for the air temperature and humidity were 
devised from linear regression analysis. MATLAB was used as the software of choice for the linear regression analysis due to its relative accuracy and speed. The differential forms of the correlations obtained are shown in Eqs. (11) - (12) and the results of the correlations are plotted in Figures 2 and 3. The $\mathrm{T}_{\mathrm{a}}$ correlation had a $\mathrm{R}^{2}$ value of 0.9818 and the humidity $(\mathrm{X})$ correlation had a $\mathrm{R}^{2}$ value of 0.9425 , which are fine for engineering accuracy. A key assumption used in this model is that the mass flowrate of water and temperature was kept constant due to a lack of plant data.

$T_{a}=$

$\left(-1.10685078180909 E-08 t^{9}\right)+\left(1.19363961132285 E-06 t^{8}\right)-$

$\left(0.000053924644264753 t^{7}\right)+\left(0.00131599104474942 t^{6}\right)-$

$\left(0.0184834485331235 t^{5}\right)+\left(0.144800483146811 t^{4}\right)-\left(0.549465822291005 t^{3}\right)+$

$\left(0.74797883067499 t^{2}\right)-(0.627515099687093 t)+15.0607284447281$

$X=$

$\left(3.28489988873192 E-12 t^{9}\right)-\left(2.66560274983221 E-10 t^{8}\right)+$

$\left(8.90840519244936 E-09 t^{7}\right)-\left(1.69852943477991 E-07 t^{6}\right)+$

$\left(2.27420240281351 E-06 t^{5}\right)-\left(0.0000232185241839378 t^{4}\right)+$

$\left(0.000151631571778955 t^{3}\right)-\left(0.0003977659593103 t^{2}\right)-$

$(0.000337926576982626 t)+0.0095662514075434$

(12)

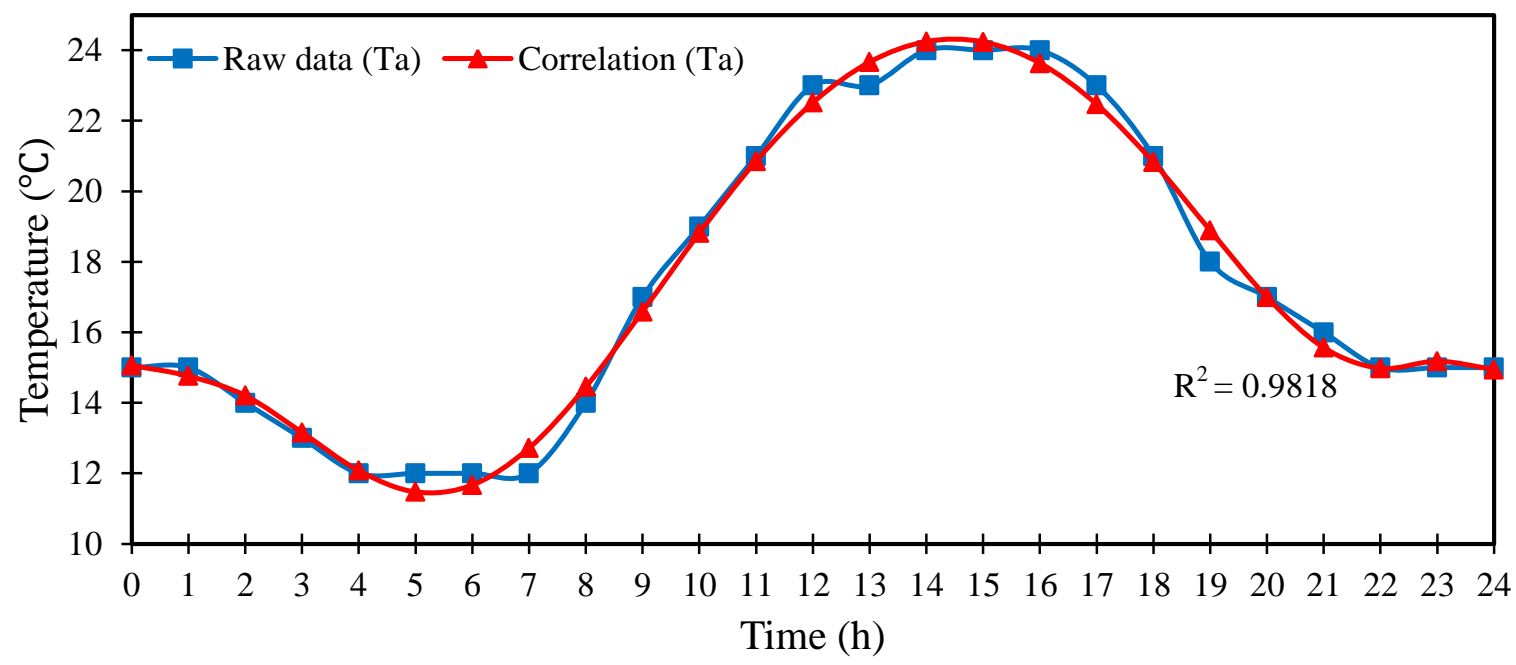

Figure 2. Comparison of correlation air temperature with raw data of Johannesburg for 24-hours 


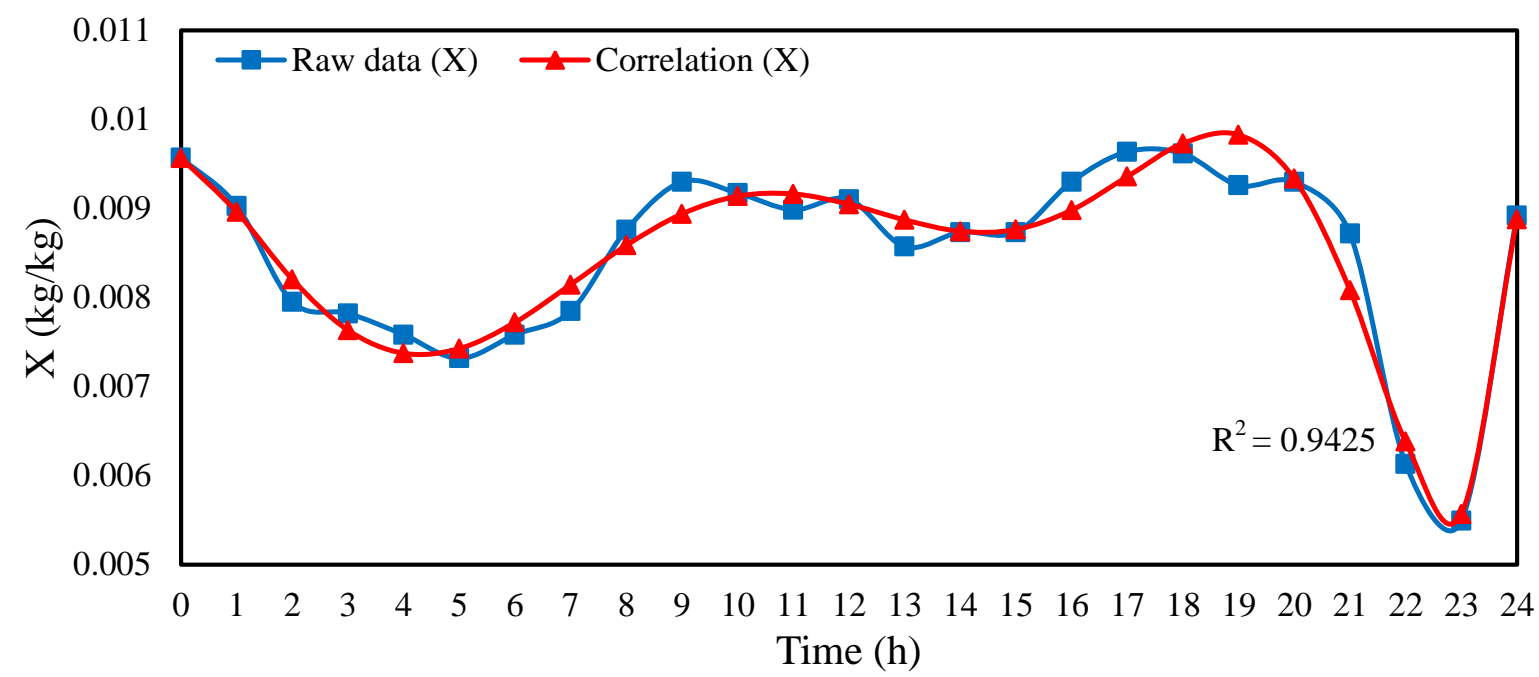

Figure 3. Comparison of correlation humidity ratio with raw data of Johannesburg for 24 hours

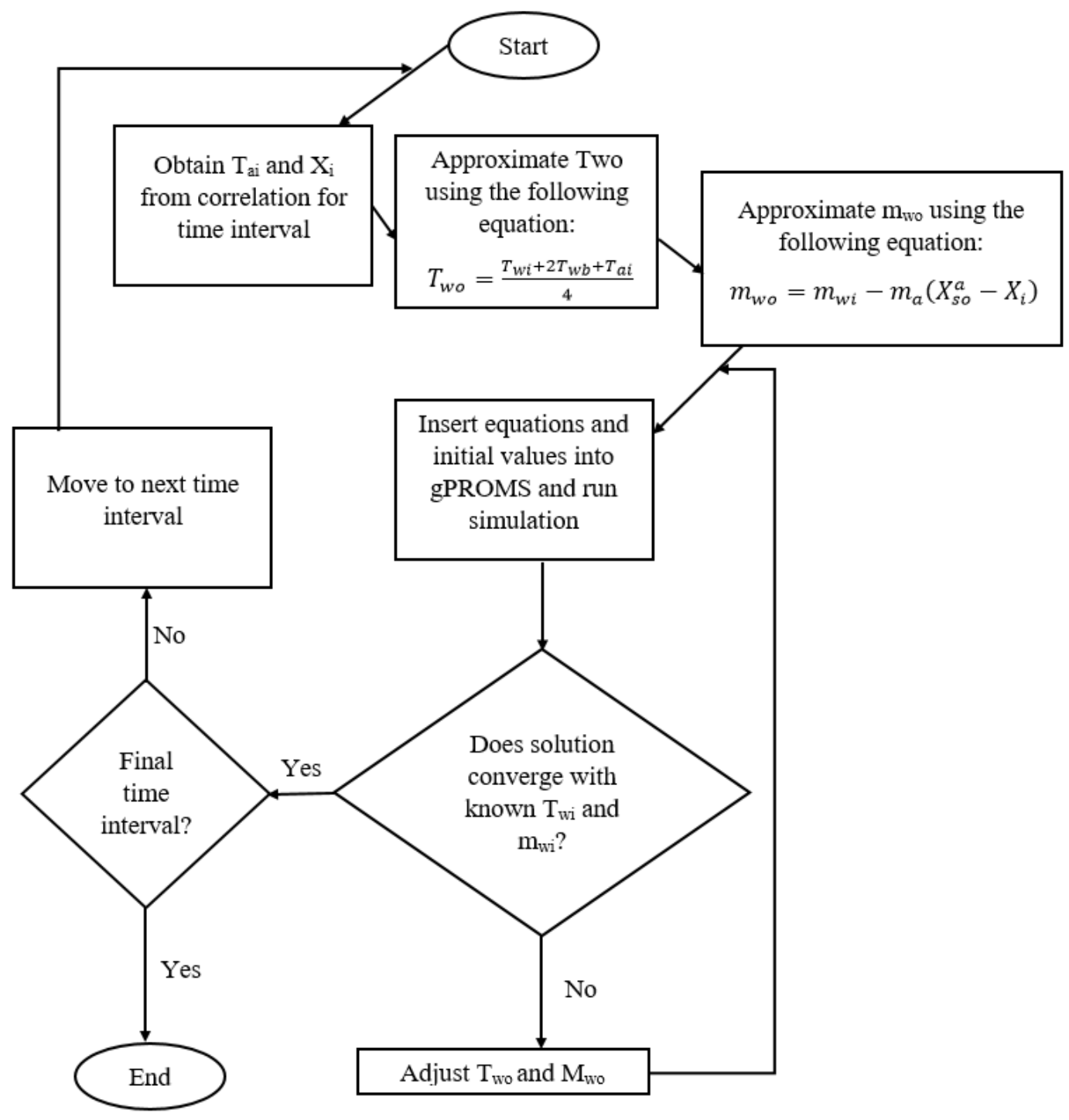




\subsection{Simulation Methodology}

Within this simulation, gPROMS was set as the software used for solving the dynamic model described earlier. The steady state model of Section 2.1 is coupled with the dynamic model of Section 2.2, resulting in a set of Differential and Algebraic Equations (DAE's)

As gPROMS will be the software used to solve the system of equations, the software would check for supersaturation at every discrete point. When the humidity of the air (X) is lower than the saturation humidity of air $\left(X_{\mathrm{s}}{ }^{\mathrm{a}}\right)$, Eqs. (1) - (5) will be used, whereas if the humidity of the air $(X)$ is greater than the saturation humidity of air $\left(X_{s}{ }^{a}\right)$, Eqs. (6) - (10) will be used. As the problem structure is a two-point boundary value problem, the system of equations had to be deconstructed and solved in a modular fashion using an iterative technique. In order to minimise the time taken to solve this set of equations, an algorithm was developed. Figure 4 shows the algorithm developed in order to solve this set of equations. Table 1 shows the specifications of the cooling tower used in the simulations. The steady-state model outlined in Section 2.1 has already been validated in the literature by Dhorat et al. (2018).

Table 1. Cooling Tower Specifications

\begin{tabular}{lc}
\hline \multicolumn{1}{c}{ Specification } & Value \\
\hline Water Inlet Mass Flowrate $\left(\mathrm{m}_{\mathrm{w.}}\right)$ & $12,500 \mathrm{~kg} / \mathrm{s}$ \\
Air Inlet Mass Flowrate $\left(\mathrm{m}_{\mathrm{a} . \mathrm{i}}\right)$ & $16,672 \mathrm{~kg} / \mathrm{s}$ \\
Height of Tower & $147 \mathrm{~m}$ \\
Height of Fill $(\mathrm{H})$ & $2.504 \mathrm{~m}$ \\
Air Inlet Diameter & $104.5 \mathrm{~m}$ \\
Air Outlet Diameter & $60.85 \mathrm{~m}$ \\
Fill Type & Splash Grid Packing \\
Water Inlet Temperature $\left(\mathrm{T}_{\mathrm{i}}\right)$ & $40{ }^{\circ} \mathrm{C}$ \\
Ambient Pressure & $84100 \mathrm{~Pa}$ \\
\hline
\end{tabular}

\subsection{Results and Discussion}

Figures $5-8$ presents the results obtained from the dynamic simulation. As there has been little work on the dynamic behaviour of cooling towers, the results obtained have shown transient behaviour, which has rarely been discussed in the literature.

One benefit of keeping the water stream temperature fixed is that the true effects of the variable air temperature and humidity can be investigated. Figure 5 shows the tower outlet 
water and air temperatures. Upon observation, it can be seen that despite the drastic change in air temperature, the outlet water temperature only changes within a range of $2{ }^{\circ} \mathrm{C}$. Therefore, it can be said that the inlet air temperature has very little effect on the outlet temperature. This analysis is indeed correct as it proves the cooling is via evaporation and not sensible heat transfer. To consolidate this statement, it can be seen that at 23:00 and 24:00 hours, the air temperature remains constant at around $15{ }^{\circ} \mathrm{C}$ but there is a $1{ }^{\circ} \mathrm{C}$ increase in the water outlet temperature, which will be explained in the next paragraph.

Figure 6 shows the distribution of the inlet and outlet humidity ratios. Generally, the distribution has remained nearly constant over the 24-hour period except between 22:00 and 23:00 hours where the relative humidity drops by $2 \%$. This $2 \%$ decrease explains why even though the temperature of the air inlet is constant, the outlet water temperature decreases by 1 ${ }^{\circ} \mathrm{C}$. As the air is drier (lower humidity ratio), there is more evaporation occurring and thus a greater degree of cooling.

Figures 7 and 8 show the outlet water mass flowrates and the mass of water being evaporated within the cooling tower. Out of all the parameters, these two parameters were found to be the most transient and this was in line with expectations. As the whole process is driven by evaporation and the humidity ratio of the air is constantly changing, the change in the outlet water flowrate would be proportional to the humidity of the inlet air. For example, between 21:00 - 23:00 hours, when there is a significant change in the humidity ratio, the outlet mass flowrate decreases by $42 \mathrm{~kg} / \mathrm{s}$. Considering the scale of the cooling tower, a decrease in the mass flowrate by $42 \mathrm{~kg} / \mathrm{s}$ is a major change, which cannot go unnoticed without further form of control.

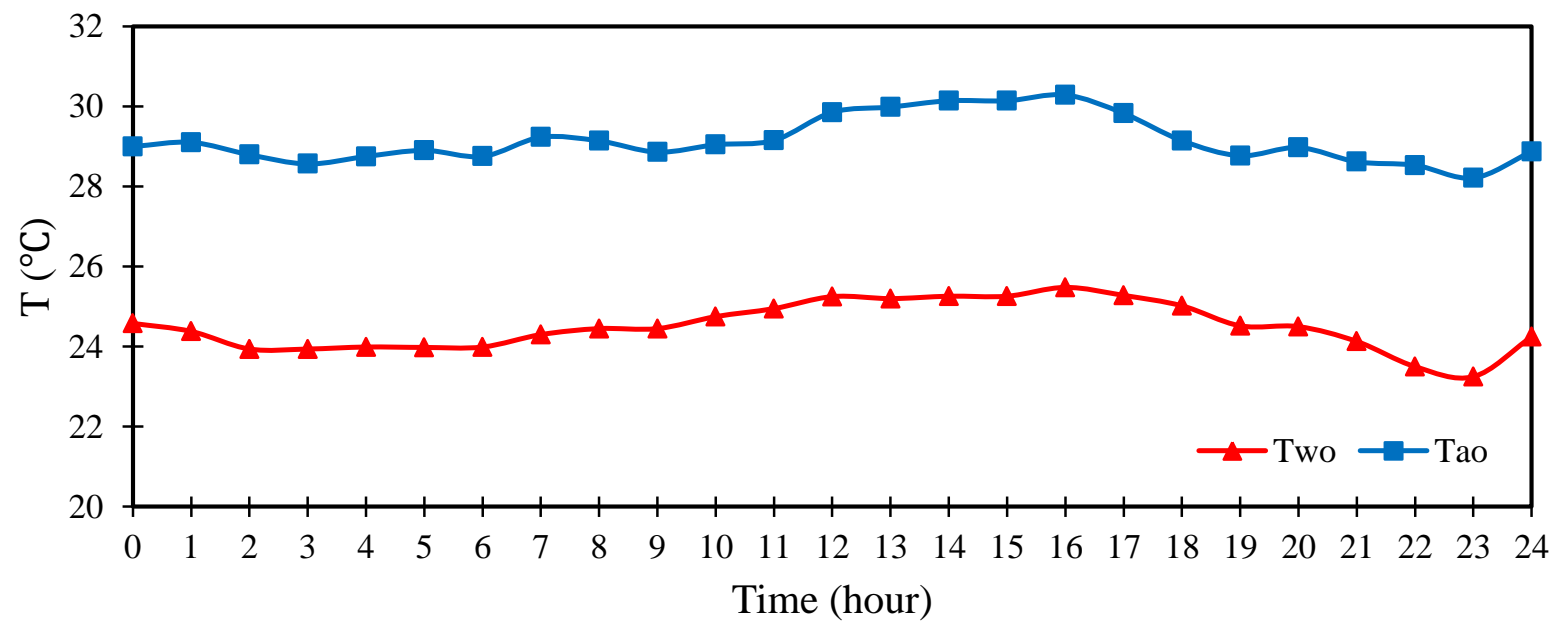

Figure 5. Dynamic model results - outlet temperature distributions over time 


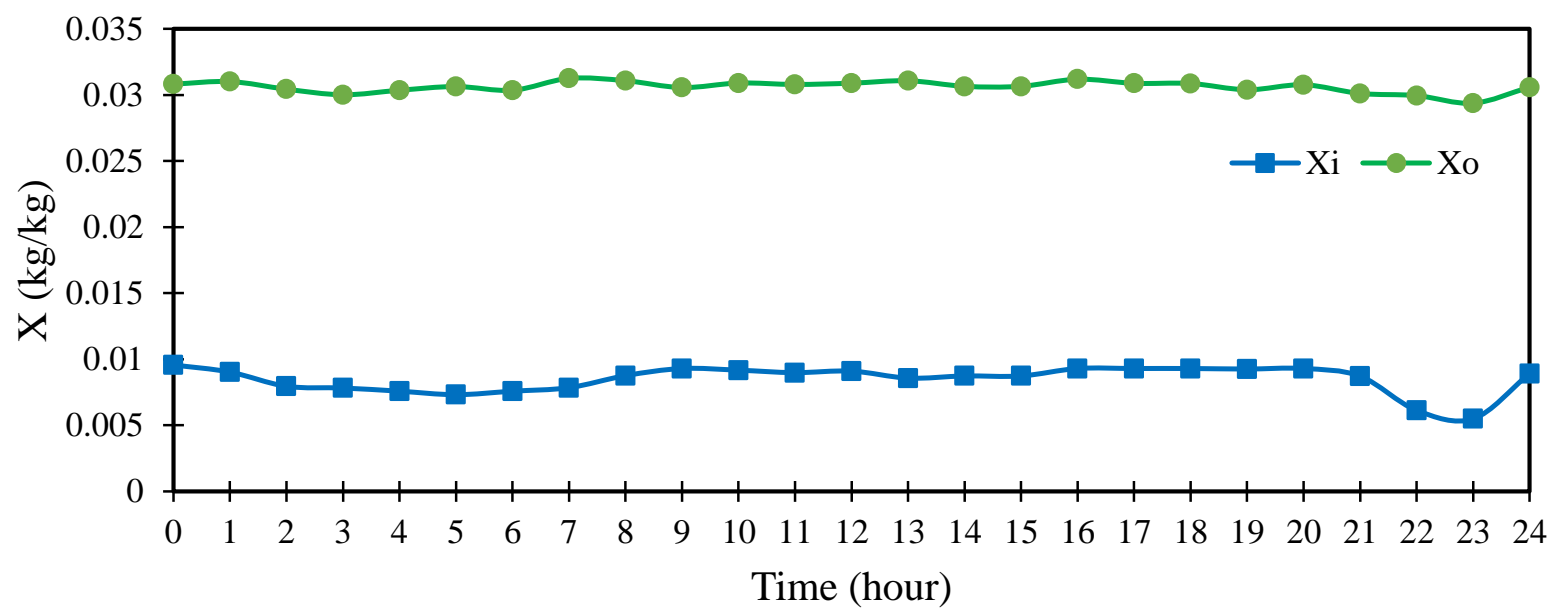

Figure 6. Dynamic model results - humidity ratio distributions over time

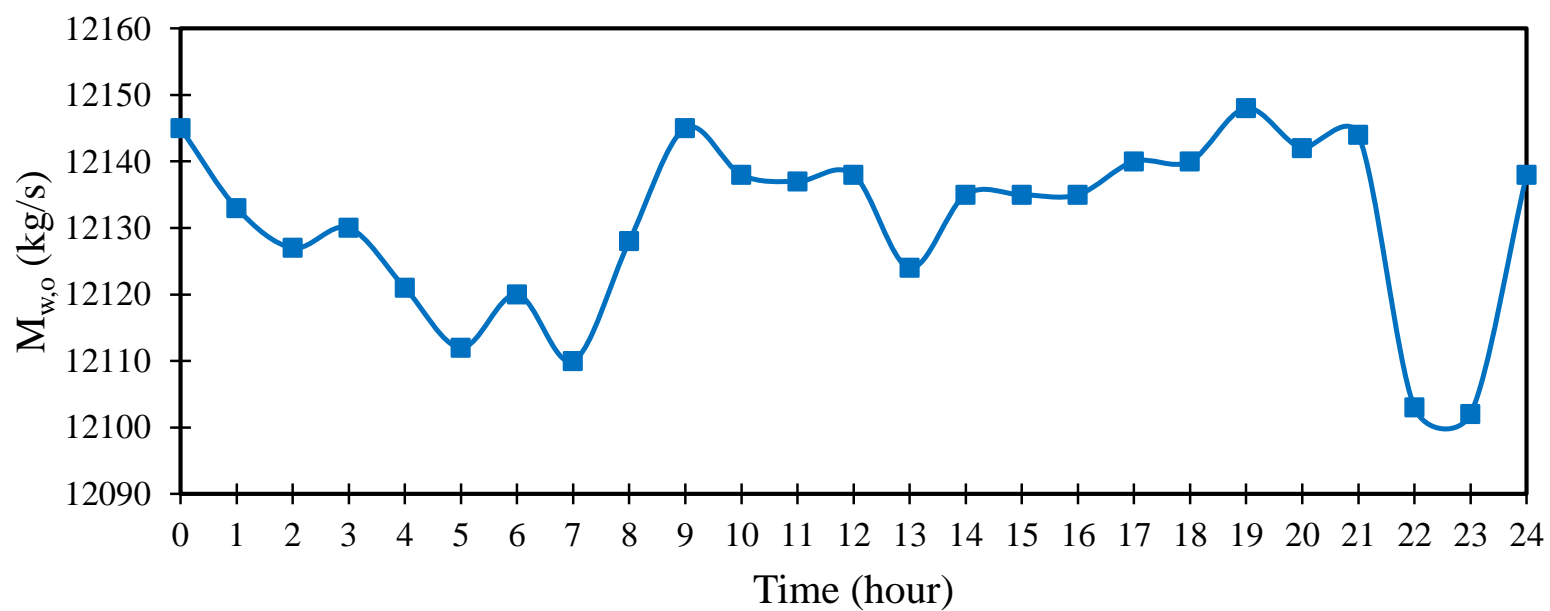

Figure 7. Dynamic model results - outlet water mass flowrate distribution over time

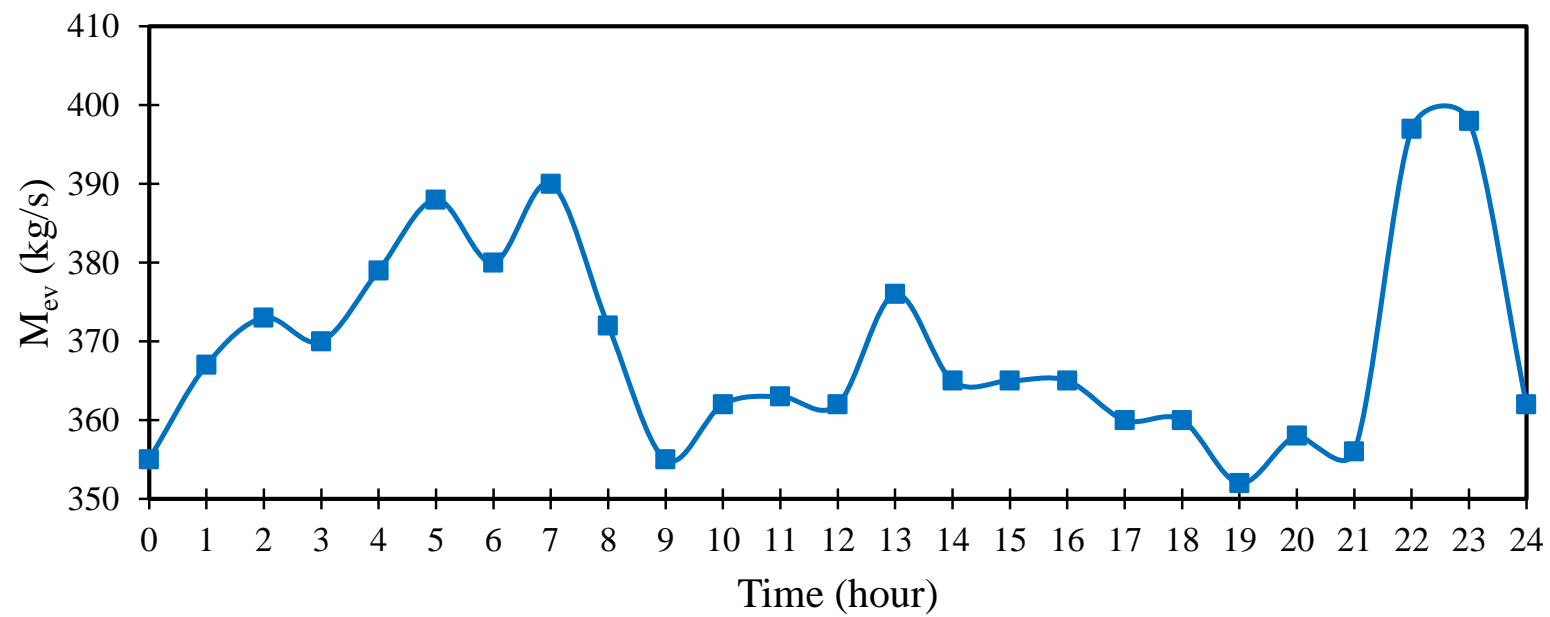

Figure 8. Dynamic model results - evaporated water mass flow distribution over time 


\section{Collection Basin Modelling}

As the process dynamics of the main cooling tower had now been successfully modelled, a model was developed for the collection basin at the bottom of a cooling tower. Upon running the model, an optimisation scheme will be set up in order to optimise the makeup water flowrate. Figure 9 shows a schematic diagram of the collection basin. As only the diameter is known from literature, the height of the collection basin is assumed to be $3 \mathrm{~m}$ due to the large amount of cooling water processed. In addition, a deep depth is required for hyperbolic cooling towers, as during shutdown; water from the process will be removed. Furthermore, it is required to provide a sufficient hydraulic head for the pump (Hensley, 1985).

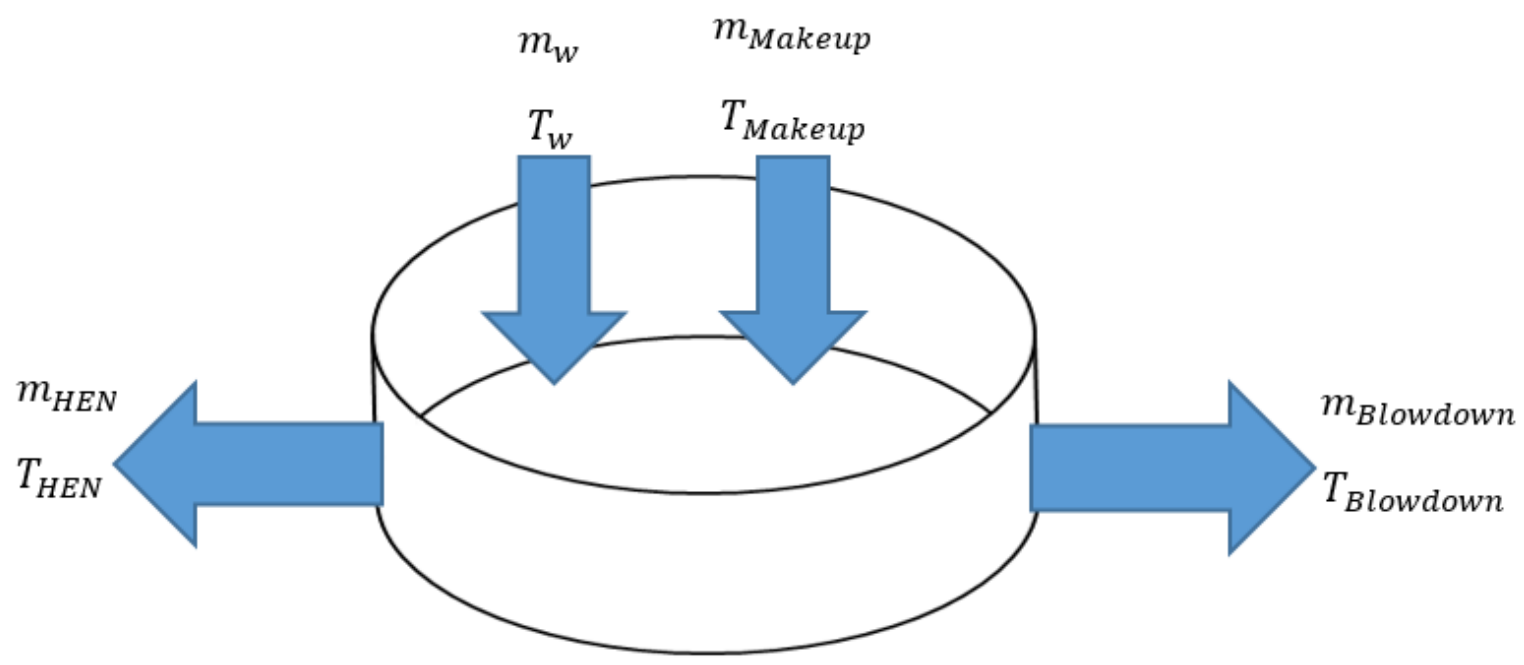

Figure 9. Schematic diagram of collection basin under investigation

The equations used for this model will developed from first principles and elementary mass balances. Accumulation within the collection basin is an elementary mass balance as shown in Eq. (13).

$\frac{d M}{d t}=\left(m_{\text {wo }}+m_{\text {Makeup }}\right)-\left(m_{\text {HEN }}+m_{\text {Blowdown }}\right)$

The mass of the water can be written in terms of conservation laws, which can lead to the average height of the water level as shown in Eq. (14).

$M=\rho V_{\text {water }}=\rho A H$

The mass of blowdown flowrate is the mass flowrate that is ejected from the system due to a build-up in concentration of contaminants, anti-scalants and antifreeze as water is evaporated. The function is expressed as shown in Eq. (15). $\mathrm{n}_{\text {cycle }}$ is the number of cycles of concentration, which depends on the risk factor of corrosion and scaling. If soft water is 
used, the number of cycles of concentration used should be kept high in order to minimise the amount of freshwater that is being used. However, if seawater is being used, the maximum value should be less than two (Buecker, 1997).

$m_{\text {Blowdown }}=\frac{M_{e}}{n_{\text {cycle }}-1}$

To obtain the mean temperature of the water within the collection basin, an elementary energy balance was undertaken, which assumed adiabatic operation from the walls of the basin as shown in Eq. (16).

$M C_{p M} \frac{d T_{M}}{d t}=$

$\left(m_{w} C_{p m_{w}}\left(T_{w}-T_{c b}\right)+\left(m_{\text {Makeup }} C_{p \text { Makeup }}\left(T_{\text {Makeup }}-T_{c b}\right)-\left(m_{H E N} C_{p H E N}\left(T_{H E N}-T_{c b}\right)-\right.\right.\right.$

$\left(m_{\text {Blowdown }} C_{\text {pBlowdown }}\left(T_{\text {Blowdown }}-T_{c b}\right)\right.$

It is assumed that the temperature of the collection basin, heat exchanger network and blowdown are the same. Therefore, Eq. (16) is reduced to Eq. (17).

$\frac{d T_{M}}{d t}=\frac{\left(m_{w} C_{p m w}\left(T_{w}-T_{c b}\right)+\left(m_{\text {makeup }} C_{\text {pmakeup }}\left(T_{\text {makeup }}-T_{c b}\right)\right.\right.}{M C_{p m}}$

The above dynamic equations for the collection basin are added to DAEs described in Section 2 for further simulation and optimisation studies described later.

As both seawater and freshwater were investigated as the cooling medium in the heat exchanger network, the assigned and initial values for both simulations are shown in Table 2. The value specified for the mass flowrates of the heat exchanger network stream, is a scaledup input value of the water going into the cooling tower $(12,500 \mathrm{~kg} / \mathrm{s})$. The mass flowrate of the makeup water was based on the average evaporation rate found in Figure 8. The diameter was known from plant data and the makeup water temperature was assumed to be the same as the air temperature. This is due to the fact that water in storage or near the surface of the sea would usually be very close to ambient temperature. The height was assumed to be $2 \mathrm{~m}$ so that there would be sufficient water to provide enough head to the pumps. The collection basin temperature was assumed to be $24{ }^{\circ} \mathrm{C}$ as the water could never reach ambient temperature. The only difference in the simulations between seawater and freshwater is that for seawater the number of cycles has been selected to be 1.5 due to mitigation of scale formation in the fill. The reason why 5 cycles of concentration is selected for freshwater is that typically in industry for soft water, a cycle of concentration range of $5-7$ is used. In this simulation, the maximum of 7 is used in order to accentuate the difference between seawater 
and freshwater. The mass flowrate of makeup water was kept the same so that the effect of cycles of concentration can be investigated.

Table 2. Initial conditions and assigned values for dynamic collection basin model - freshwater and seawater mediums

\begin{tabular}{cccc}
\hline \multicolumn{3}{c}{ Assigned Values } \\
\hline Freshwater (Base Case A) & \multicolumn{2}{c}{ Seawater (Base Case B) } \\
\hline Parameter & Value & Parameter & Value \\
\hline $\mathrm{m}_{\text {makeup }}$ & $4,086,000 \mathrm{~kg} / \mathrm{h}$ & $\mathrm{m}_{\text {makeup }}$ & $4,086,000 \mathrm{~kg} / \mathrm{hr}$ \\
$\mathrm{m}_{\text {hen }}$ & $45,000,000 \mathrm{~kg} / \mathrm{h}$ & $\mathrm{m}_{\text {hen }}$ & $45,000,000 \mathrm{~kg} / \mathrm{h}$ \\
$\mathrm{n}_{\text {cycle }}$ & 7 & $\mathrm{n}_{\text {cycle }}$ & 1.5 \\
$\mathrm{D}$ & $104.5 \mathrm{~m}$ & $\mathrm{D}$ & $104.5 \mathrm{~m}$ \\
$\rho_{\text {water }}$ & $1000 \mathrm{~kg} / \mathrm{m}^{3}$ & $\rho_{\text {water }}$ & $1029 \mathrm{~kg} / \mathrm{m}^{3}$ \\
$\mathrm{~T}_{\text {makeup }}$ & $16.1^{\circ} \mathrm{C}$ & $\mathrm{T}_{\text {makeup }}$ & $16.1^{\circ} \mathrm{C}$ \\
\hline $\mathrm{H}$ & Initial Conditions & $2 \mathrm{~m}$ \\
\hline $\mathrm{T}_{\mathrm{cb}}$ & $2 \mathrm{~m}$ & $\mathrm{H}$ & $24^{\circ} \mathrm{C}$ \\
\hline
\end{tabular}

\subsection{Results and Discussion}

Figures 10 - 13 shows the results obtained from the simulation of the dynamic collection basin model. Figure 10 shows that the level of the seawater medium rises over the period of 24 hours by $0.25 \mathrm{~m}$ (approximately $12.6 \%$ ) without any form of control. This is expected as the mass flowrate of the makeup water was fixed at $4,086,000 \mathrm{~kg} / \mathrm{h}$ and was invariant with respect to other variables. However, such a large increase in the height of the water level over a period of 24 hours is a major concern due to the long-term implications. In contrast, the freshwater simulation had yielded results, which were in line with expectations. As the flowrate of the makeup water was the same and the number of cycles of concentration had increased, there was less water leaving as blowdown water. Therefore, significant holdup was expected, and results corroborated these statements. As the basin is only $3 \mathrm{~m}$ tall, this simulation would eventually violate the height without any form of further control. Therefore, an optimisation scheme for the makeup water and number of cycles would be formulated in the next section.

Figure 11 shows the mass of water in the seawater and freshwater simulations being accumulated within the collection basin over the 24-hours. The results demonstrate that without adequate control, it would be possible to increase the mass of water in the seawater case by $11.2 \%$, resulting in extra pumping costs and further risk of fouling deposition at the surface and at the bottom of the basin. Results of the freshwater case were exponentially higher than that of the seawater due to the same reasons explained as above. 
Interestingly, Figure 12 shows the mass of blowdown water was $50 \%$ higher than the mass flowrate of the evaporated water in the seawater case. As the number of cycles of concentration was fixed at 1.5 due to the use of seawater, such a large quantity being discharged was in line with industrial standards. As scaling of equipment such as heat exchangers are to be kept to a minimum, a low number of cycles is required in order to mitigate these issues. Whereas the blowdown for the freshwater case was significantly lower than that of the seawater case as there is a lower concentration of anti-scalants and anti-freeze in the water.

Figure 13 shows the mean temperature distribution within the collection basin for both the seawater and freshwater cases. The thermophysical properties of both cooling mediums were assumed to be the same and thus the temperature did not change. As the temperature does not wildly fluctuate as it stays between $22-25{ }^{\circ} \mathrm{C}$, this is not of a major concern for the plant operator. However, this temperature must be carefully monitored, as it is the water from the collection basin, which goes through to the cooling network of the power plant.

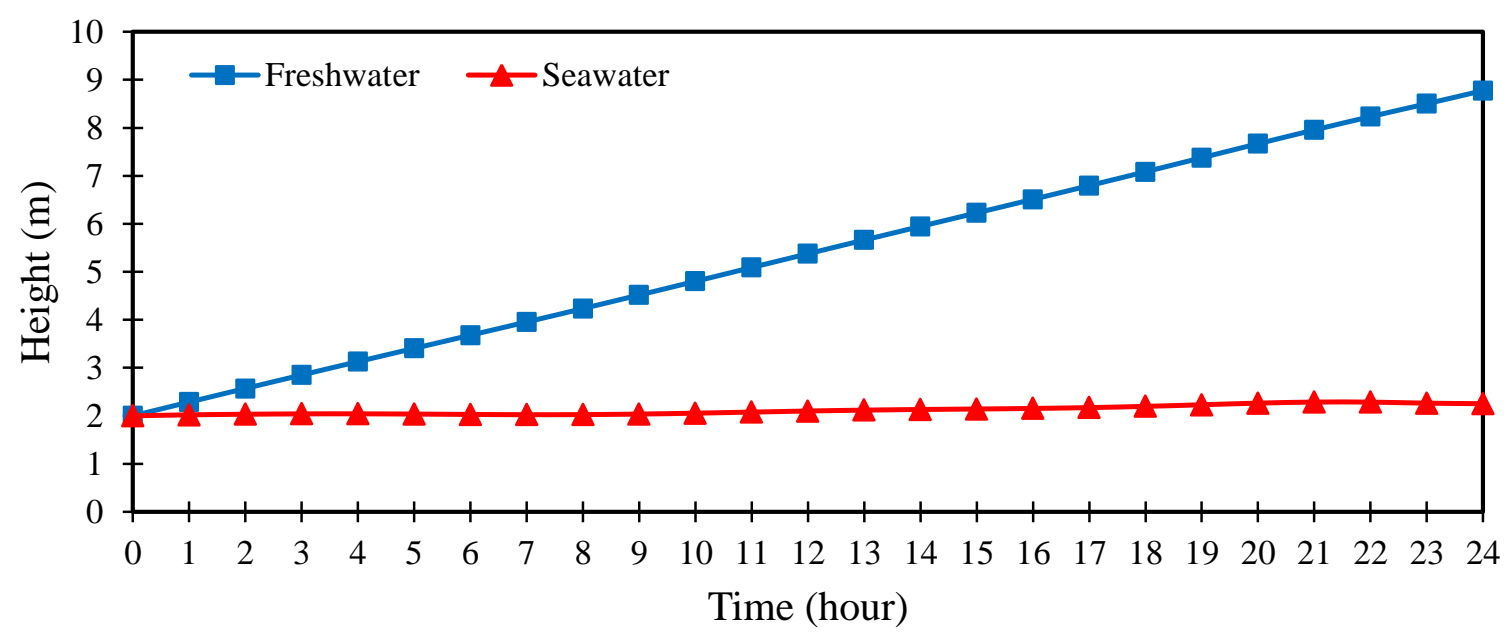

Figure 10. Collection basin model - height of water levels 


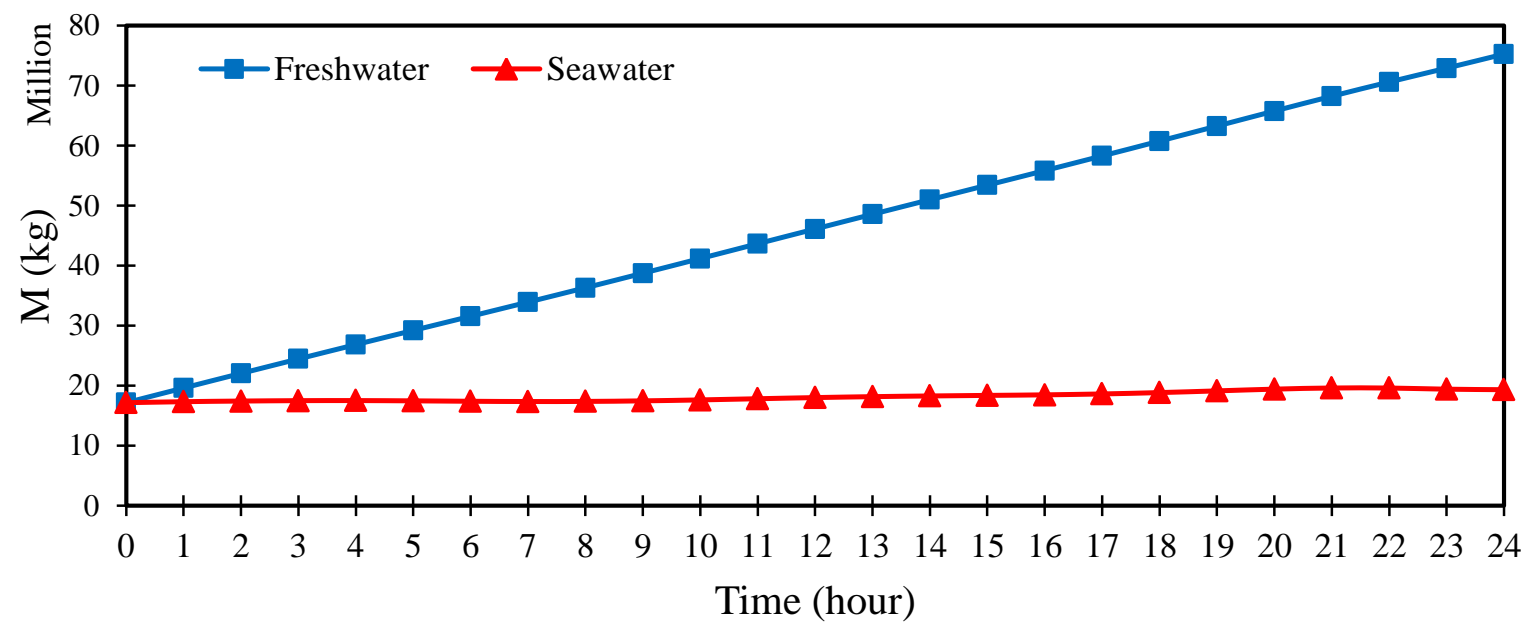

Figure 11. Collection basin model - accumulated mass of water

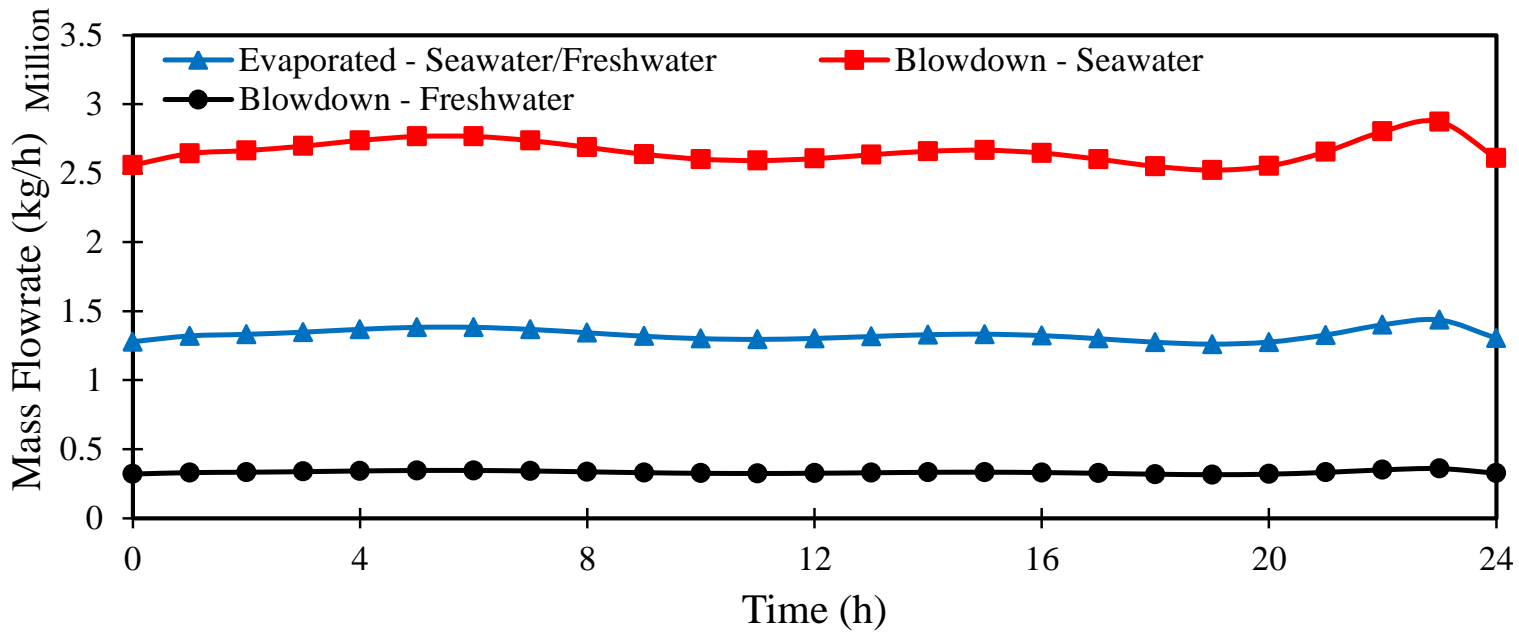

Figure 12. Collection basin model - mass flowrates of evaporated water and blowdown

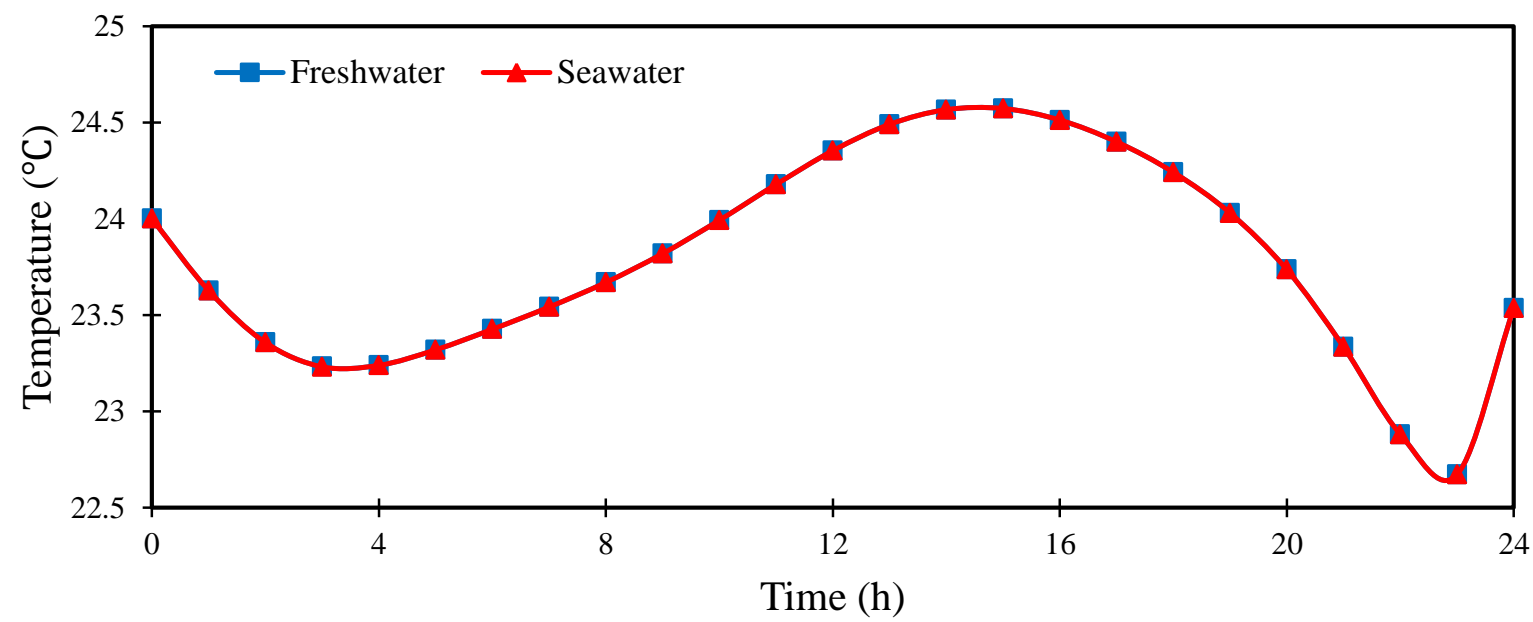

Figure 13. Collection basin model - mean temperature of water basin 


\section{Optimisation of Collection Basin}

It can be seen in Section 3 that the height of the water level is continually increasing over time. This is due to a fixed makeup rate and number of cycles within the basin and cooling tower. In terms of cost, this kind of setup can be prohibitively expensive due to excess water being pumped into the system. In this section, the operating cost per hour will be investigated through an optimisation study. As there are two potential cooling mediums for a heat exchanger network - freshwater and seawater, the effect of utilising both will be investigated. The results obtained for freshwater in Section 3 will be called Base Case A and the results obtained for seawater (Section 3) will be referred to as Base Case B. In order to compute the operating cost of the collection basin, an adapted function from Panjeshahi et al. (2009) was used as shown in Eq. (18). This function also includes the latest chemical engineering plant cost index (CEPCI) for June 2017.

Operating cost $=$ pumping cost + makeup cost + chemical treatment cost + blowdown treatment cost

$O C=(2.99 E-04 \times P P)+\left(1.3693 E 02 \times m_{\text {makeup }}\right)+\left(0.28322 \times m_{w}\right)+(0.14166 \times$

$m_{\text {blowdown }}$ )

Where PP is known as the total pumping power in $\mathrm{kW}$. Eq. (19) shows the equation used within the simulation to calculate the pumping power. The pumping power is also known as the shaft power and is a function of the total head required along with the mass flowrate and density of the water. In this study, the efficiency $(\eta)$ will be fixed at 0.85 because larger pumps tend to have a higher degree of efficiency. The subscript i refers to the mass flowrates of makeup water, heat exchanger network and blowdown. The pumping power of the inlet water from the cooling tower was not considered, as it is not part of the collection basin as it utilises gravity. The total head was also selected to be $5 \mathrm{~m}$ across all four streams.

$P P=\sum 0.746 \frac{\frac{m_{i}}{1000} \rho g h}{3.6 \times 10^{6} \eta} ; \quad i=$ Makeup, HEN,Blowdown

\subsection{Optimisation Methodology}

The software used for the optimisation is gPROMS, which has a single shooting solver. The software allows the user to undertake steady state or dynamic optimisation. The program is chosen based on the merit that the solution algorithm does not have to be written but only 
defined. It also has the flexibility to select a single model for different optimisation purposes. In this work, the optimisation will solely be based upon nonlinear programming (NLP), which will utilise a successive quadratic programming (SQP) method for solving.

\subsection{Problem Description}

The objective of this optimisation work is to find the optimal hourly cost of the collection basin over a period of 24 hours. The first objective function for both freshwater and seawater is to minimise the hourly operating cost over the specified time. Thereafter the mass of accumulated water and the mass flow of makeup water will be minimised.

For each optimisation case study, the parameters that will be optimised are the mass flow of makeup water and the number of cycles of concentration. The constraints for the mass flow of makeup water will be kept between $0 \mathrm{~kg} / \mathrm{hr}$ and 2 million $\mathrm{kg} / \mathrm{hr}$ for freshwater. For seawater, this constraint would be between $0 \mathrm{~kg} / \mathrm{hr}$ and 4 million $\mathrm{kg} / \mathrm{hr}$. This constraint is sensible as Base Case A shows a mass flow of 4.08 million $\mathrm{kg} / \mathrm{hr}$ and this study focuses on the minimisation of this variable across all the case studies. The constraint of the number of cycles will depend on the cooling tower medium. As previously mentioned, for freshwater use, the number of cycles can vary between 5 and 7 cycles. Whereas for seawater systems, due to a higher rate of solid deposition in the system, the value is constrained between 1.1 and 2.0.

An equality endpoint constraint was also introduced into the system as a form of control so that the height of the water will return to $2 \mathrm{~m}$ after the 24-hour period. By maintaining a final height of $2 \mathrm{~m}$, it would be possible for the system to accommodate for fluctuations in the rate of accumulation throughout the course of the day.

The generic form of the NLP is descriptively outlined below (PSE, 2004):

Objective function:

$$
\begin{aligned}
& \min \text { OR max } z\left(t_{f}\right) \\
& t_{f}, v, u(t), t \in\left[0, t_{f}\right]
\end{aligned}
$$

Subject to:

Process model:

$$
f(x(t), \dot{x}(t), u(t), v)=0 \quad t \in\left[0, t_{f}\right]
$$

Initial conditions:

$$
I(x(0), \dot{x}(0), u(0), v)=0
$$

Time horizon bounds:

$$
t_{f}^{\min } \leq t_{f} \leq t_{f}^{\max }
$$

Control variable bounds:

$$
u^{\min } \leq u(t) \leq u^{\max }
$$$$
t \in\left[0, t_{f}\right]
$$ 
Time invariant parameter bounds: $\quad v^{\min } \leq v \leq v^{\max }$

Endpoint constraints:

$$
\begin{aligned}
& w\left(t_{f}\right)=w^{*} \\
& w^{\min } \leq w\left(t_{f}\right) \leq w^{\max }
\end{aligned}
$$

The process model is the full model written into a compact form $f(x(t), \dot{x}(t), u(t), v)=0$.

Where $t$ is the time horizon or independent variable, $v$ is the time invariant parameters, $x(t)$ is the full set of differential-algebraic equations (DAE), $\dot{x}(t)$ is the derivatives with respect to time and $u(t)$ is the control variables. $w^{*}$ is an endpoint equality constraint where the value at the final time horizon must satisfy this constraint. $w$ is an inequality endpoint constraint where the value at the final time interval must fall within a specified range. The objective function refers to the variable $z\left(t_{f}\right)$ being minimised or maximised as required. In this study, the time horizon will be fixed from $0-24$ hours as the optimisation is focused on hourly operating cost.

In Sections 4.3 and 4.4 , the nonlinear optimisation problem is mathematically expressed as:

Objective function: $\quad$ Outlined in Sections 4.3 and 4.4

Subject to:

Process model: $\quad f(x(t), \dot{x}(t), y(t), u(t), v)=0 \quad t \in[0,24]$

Initial conditions: $\quad I(x(0), \dot{x}(0), y(0), u(0), v)=0$

Time horizon bounds: $\quad t_{f}=24$

Piecewise constant control bounds: Outlined in Sections 4.3 and 4.4

Equality endpoint constraints: $\quad H\left(t_{f}\right)=2 \mathrm{~m}$

\subsection{Optimisation using Freshwater Cooling Medium (Cases 1-3)}

The objective functions for the three cases are shown in Table 3 along with the piecewise constant controls.

Table 3. Objective functions and piecewise controls for freshwater optimisation

\begin{tabular}{c|c|c|c}
\hline & Case Study 1 & Case Study 2 & Case Study 3 \\
\hline Objective Function & Minimise operating cost & $\begin{array}{c}\text { Minimise accumulation } \\
\text { (holdup) of water in tank }\end{array}$ & $\begin{array}{c}\text { Minimise makeup water } \\
\text { flowrate }\end{array}$ \\
\hline Piecewise Constant & \multicolumn{2}{|c}{$0^{\text {min }} \leq m_{\text {makeup }} \leq 2 E 06^{\text {max }}$} \\
Controls & \multicolumn{3}{c}{$1.1^{\text {min }} \leq n_{\text {cycle }} \leq 2^{\text {max }}$} \\
\hline
\end{tabular}




\subsubsection{Freshwater Optimisation Results}

The optimisation results of cases $1-3$ are shown in in Figures $14-17$. Analysis of the operational cost in Figure 14 shows that the average cost of case 1 is $38.88 \%$ cheaper over the 24-hour period compared to Base Case A. The points after 3 hours for Base Case A are not shown in Figure 14 as they go beyond 3m, which is not in control. Figure 10 shows the full data for Base Case A over the 24-hour period. Case 2 shows that the operating costs are reduced by $40.25 \%$ compared to that of Base Case A. This was expected, as there is no more excess water in the system as the flowrate of the makeup water shown in Figure 16 is kept at a minimum of 1.37 million $\mathrm{kg} / \mathrm{h}$. By minimising the makeup water, there are less chemical treatment costs and blowdown water treatment costs. With respect to case 3 , it can be seen that the average cost over the 24-hour period was cheaper than Base Case A by $39.46 \%$. It can also be seen that the average cost is lower than case 1 by $0.95 \%$. This is an interesting observation as case 3 had used $1.56 \%$ less make up water in comparison to case 1 over the 24-hour period. Therefore, it can be said that the mass flow of makeup water is the biggest factor in dictating the operating cost.

Figure 15 shows the variation in the height of water over the 24-hour period. In comparison to Base Case A where the final height ended at 9 case 1 had yielded a final height of $2 \mathrm{~m}$, which means that the solution is feasible. By turning off the makeup water at 23:00, the mass of water accumulated had reduced as shown by the height dropping from $2.3 \mathrm{~m}$ to $2 \mathrm{~m}$. Controlling the level in this way has many implications such as finer control of the final height at the end of the day and maintenance of the makeup water pump can be undertaken. Due to the variable nature of freshwater mineral content, this hour can be used for a laboratory specified means of the dissolved solids content so chemical treatment of the water can be adjusted. In comparison to Base Case A and case 1, the height in case 2 fluctuates between $1.97 \mathrm{~m}$ and $2.02 \mathrm{~m}$, which shows a better degree of control as the mass of water accumulated within the basin has been minimised. Results for case 3 shows results which are in contrast to Base Case A and cases 1 and 2. It can be seen that there are several points where the height peaks before reducing due to a reduction in the makeup water flowrate. These results are feasible due to the height not increasing more than $3 \mathrm{~m}$ and the end height was still found to be $2 \mathrm{~m}$.

Figure 16 shows the makeup water flowrate over the 24 -hour period. It is seen that case 1 had reduced the flowrate of water on average by $57.80 \%$, which represents a significant 
amount of water being saved in comparison to Base Case A. By turning off the makeup water flowrate between 23:00 and 24:00, it yields significant savings with respect to pumping costs as well as giving a window of time for basic maintenance of the pumps. With respect to case 2 , it can be seen it has the lowest makeup flowrate over the 24 hours. As a result had a $61.55 \%$ decrease in makeup water costs - the highest difference between the three cases. For case 3 , it can be seen that the pump essentially runs at two speeds; 2 million $\mathrm{kg} / \mathrm{hr}$ and 1.37 million $\mathrm{kg} / \mathrm{hr}$. This configuration is feasible, as a two-speed pump would yield significant savings over a single speed pump. Although variable speed pumps are the industrial norm, the two-speed pump would yield a lower capital cost as well as it being a proven technology with a higher mean time between failures (MTTF).

Figure 17 shows how the number of cycles is affected with time and results show significant variation within the 24-hour period. In terms of case 1 and 2, the number of cycles is very similar, and this is because as the operating cost and water accumulations are the objective functions, it is the amount of water within the tower basin which dictates the overall operating cost. In comparison to Base Case A, cases 1 and 2 turn off the pumps within the final hour and increases the number of cycles in order to conserve and minimise water usage. With respect to case 3, in comparison to Base Case A, the value starts higher at 7 before returning to 5. This is because with higher cycles of concentration, the amount of water being accumulated also begins to increase. By using the number of cycles as a control variable, it is possible to control the holdup in the system so that the final endpoint criterion of $2 \mathrm{~m}$ is fulfilled.

The average power required by the pumps for blowdown and makeup water are shown in Table 4. For case 1, Results show that the pump required for the makeup water stream has now decreased by $60.39 \%$, which are in line with expectations. As the makeup water flowrate has now been optimised, the amount of excess water within the collection basin has now decreased and therefore the power required by the pump has decreased. The pump power requirement of the blowdown stream has also decreased by $4.30 \%$. In the case of case 2 , the pumping power required by the makeup water stream has decreased by $61.55 \%$, which is a further $1.16 \%$ decrease in comparison to case 1 . The reason for this difference is due to less makeup water being utilised as well as a significant amount reduction in blowdown water $(28.06 \%)$. As there is less water being expelled through blowdown, the makeup requirements also reduce by the same ratio. In the instance of case 3 , the pumping requirements by the system does not reduce as much as cases 1 and 2. Although there is a reduction in blowdown by $2.38 \%$, this is not as much of a significant decrease due to the fact that the number of 
cycles reduce throughout the day. This reduction in the number of cycles is purely due to control as the collection basin has to meet the $2 \mathrm{~m}$ level constraint at the end of the 24 hours. As the system tries to meet this constraint, the mass of water for blowdown increases and thus incurs an extra cost.

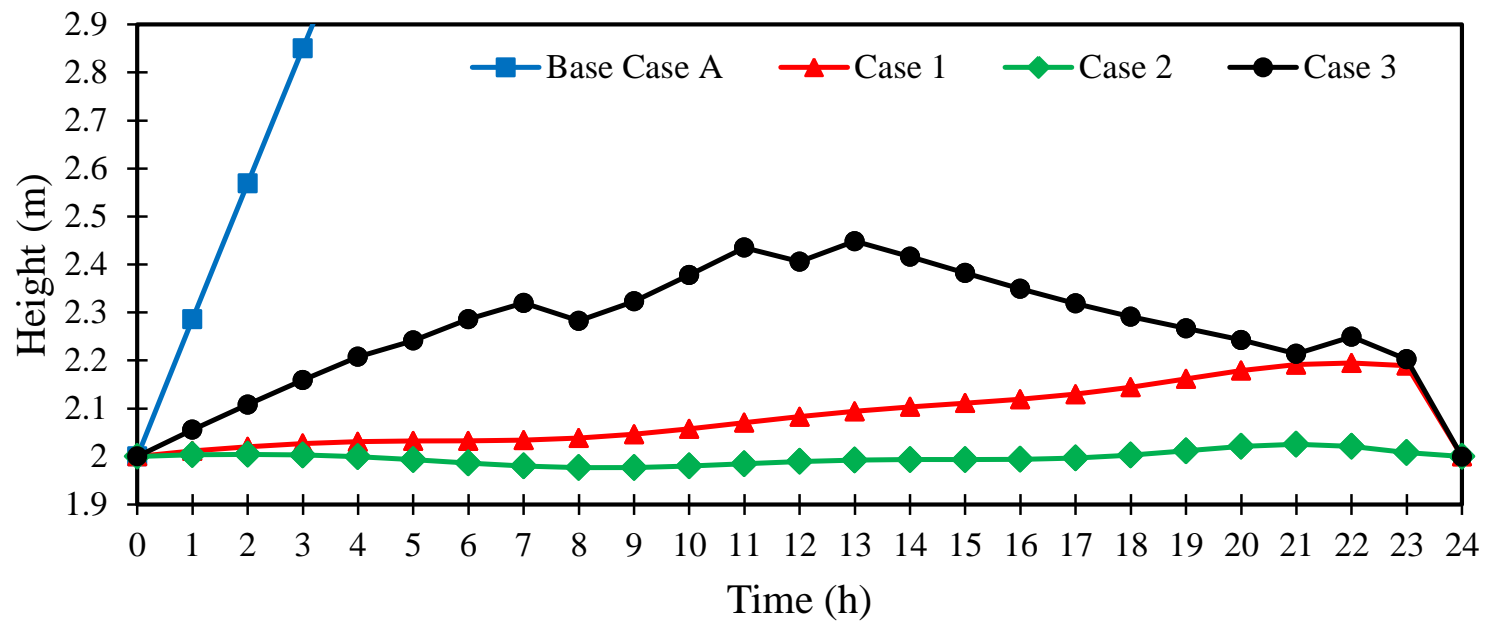

Figure 14. Freshwater medium optimisation - height

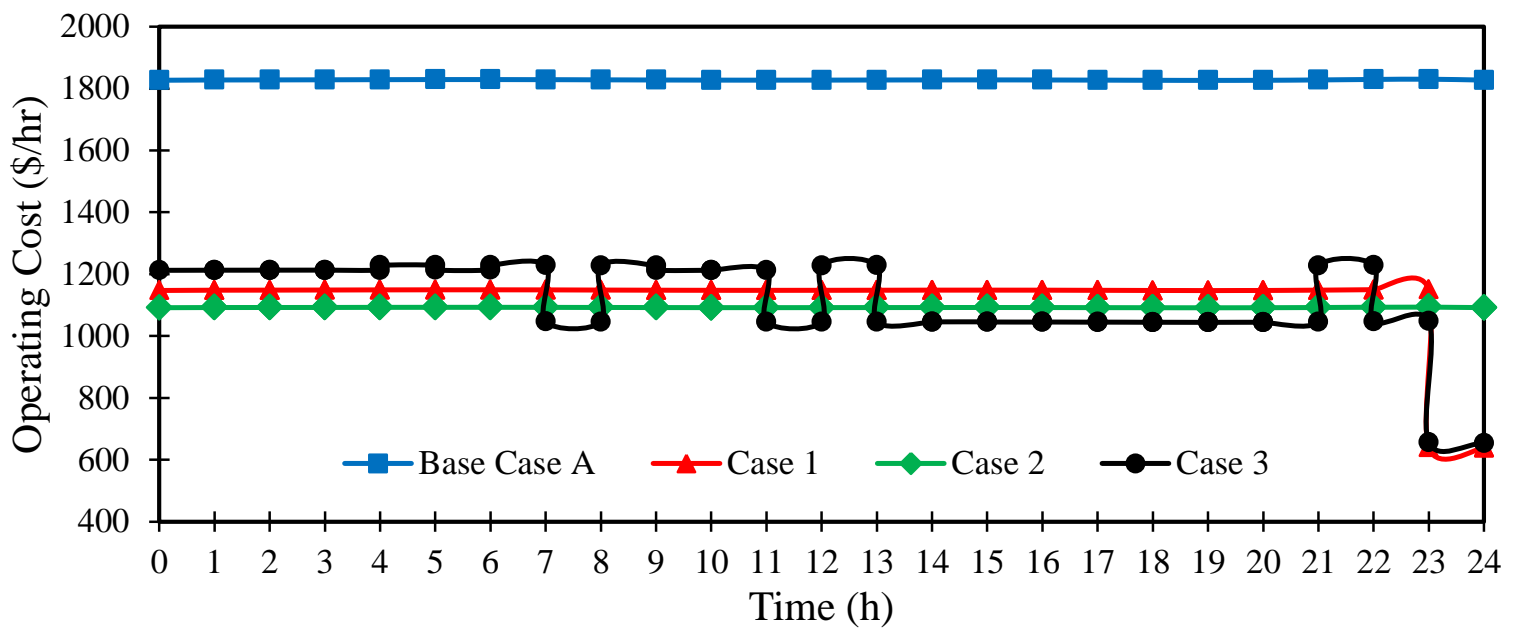

Figure 15. Freshwater medium optimisation - operating cost 


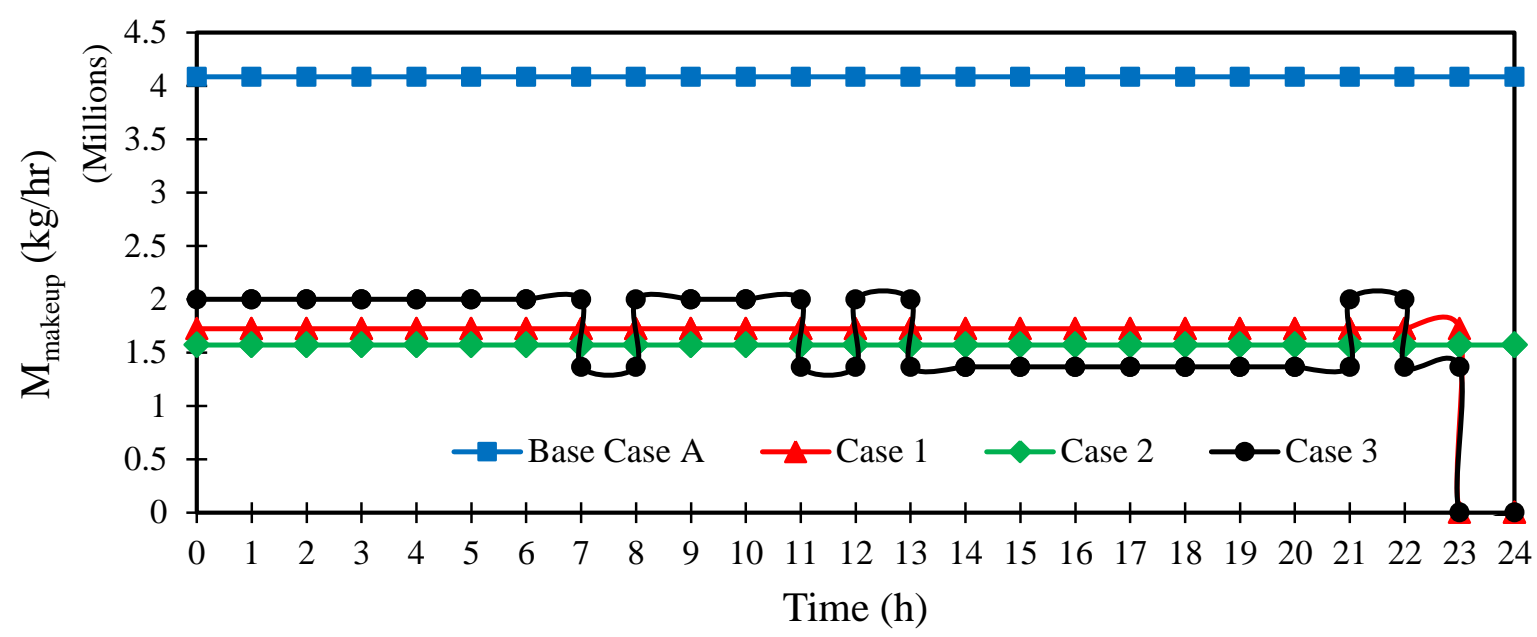

Figure 16. Freshwater medium optimisation - mass flow of makeup water

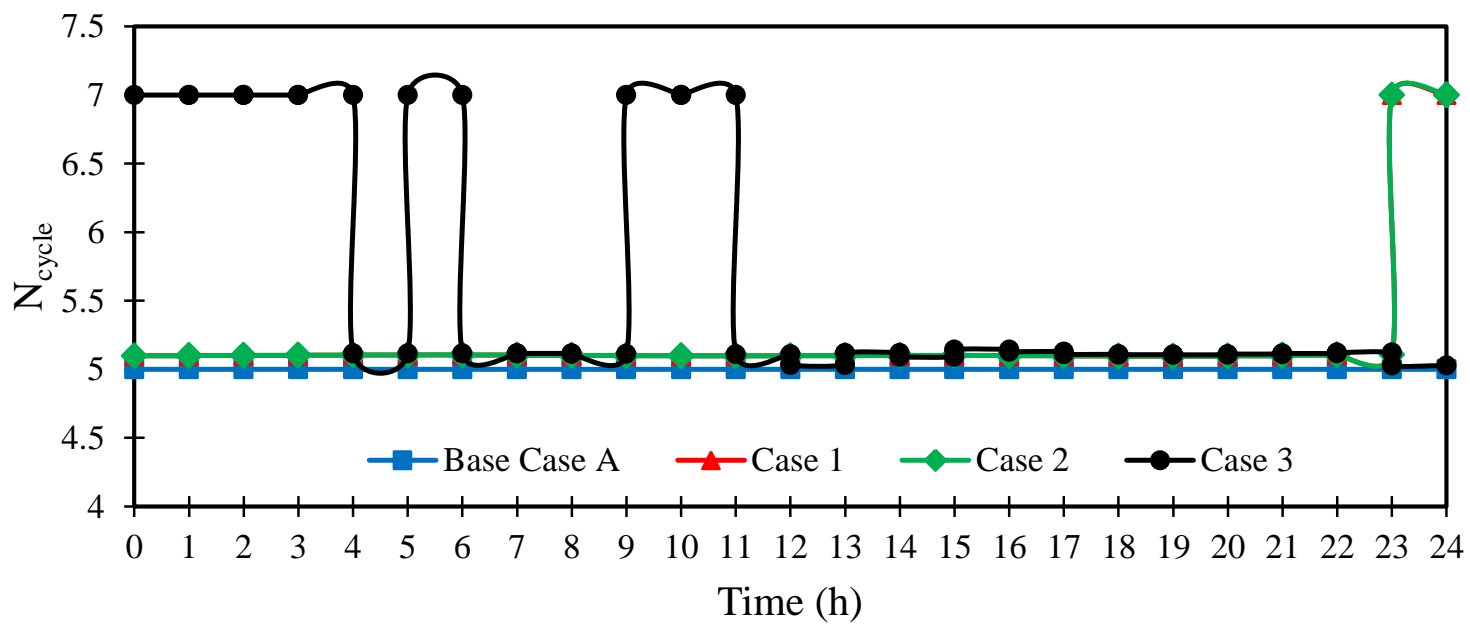

Figure 17. Freshwater medium optimisation - Number of cycles

Table 4. Optimised average pump requirements and costs for cases 1, 2 and 3

\begin{tabular}{|c|c|c|c|c|c|c|}
\hline & \multicolumn{6}{|c|}{ Average Pumping Power (kW) } \\
\hline & \multicolumn{2}{|c|}{ Case 1} & \multicolumn{2}{|c|}{ Case 2} & \multicolumn{2}{|c|}{ Case 3} \\
\hline & Blowdown & Makeup & Blowdown & Makeup & Blowdown & Makeup \\
\hline Base Case A & 3.97 & 48.84 & 3.97 & 48.84 & 3.97 & 48.84 \\
\hline Optimised & 3.80 & 19.35 & 2.86 & 18.78 & 3.88 & 19.80 \\
\hline$\%$ Change & -4.30 & -60.39 & -28.06 & -61.55 & -2.38 & -59.46 \\
\hline
\end{tabular}




\subsection{Optimisation using Seawater Cooling Medium (Cases 4-6)}

In this section, the seawater cooling medium is to be utilised and an optimisations scheme for three different objective functions is to be undertaken. Table 5 shows the objective functions to be used in this work.

Table 5: Objective functions of optimisation using seawater cooling medium

\begin{tabular}{c|c|c|c}
\hline & Case Study 4 & Case Study 5 & Case Study 6 \\
\hline Objective Function & Minimise operating cost & $\begin{array}{c}\text { Minimise accumulation } \\
\text { (holdup) of water in tank }\end{array}$ & $\begin{array}{c}\text { Minimise makeup water } \\
\text { flowrate }\end{array}$ \\
\hline Piecewise Constant & \multicolumn{3}{|c}{$0^{\text {min }} \leq m_{\text {makeup }} \leq 4 E 06^{\text {max }}$} \\
Controls & \multicolumn{3}{c}{$1.1^{\text {min }} \leq n_{\text {cycle }} \leq 2^{\text {max }}$} \\
\hline
\end{tabular}

\subsubsection{Seawater Optimisation Results}

Figure 18 shows the optimised results of the distribution of the height of the water. It can be seen that the results for cases 4 and 6 draw parallel to the results obtained for freshwater. This was expected, as the simulation is the same except for the fact that the number of cycles are significantly lower. Another reason why the similarities had occurred is due to the fact that the operating cost is directly proportional to the makeup water flowrate. With an increase in the makeup flowrate, pumping costs and the chemical treatment costs would increase proportionately. With respect to case 5, the range of the height was found to between $1.95 \mathrm{~m}$ and $2.05 \mathrm{~m}$, which means that this process is very well controlled in comparison to cases 4 and 6 in which there was a significant deviation from the required height of $2 \mathrm{~m}$.

The optimised operating cost obtained in Figure 19 shows that the distribution of the values for cases 4 and 5 are very similar to those in Section 4.3. However, the actual costs themselves are significantly higher than those found for freshwater. This is expected due to reasons outlined in the previous paragraph with the addition that more seawater is expunged due to a lower number of cycles of concentration. In comparison to Base Case B, case 4 yields a $29.8 \%$ decrease in cost. This is largely down to a decrease in makeup water flowrate as shown in Figure 20. With respect to the operating cost of case 5, it can be seen that the optimised case yields an average saving of $21.24 \%$ compared to Base Case B. Such a large percentage is a significant result as over the course of a year, there can be a substantial saving made with proper optimisation of the operating parameters. The range of the hourly cost was between $\$ 1743$ and $\$ 1775$ per hour, which is a $1.8 \%$ difference between the maximum and 
minimum. Considering existing literature up until now (10/07/2017), has only focused on predicting the operating cost of cooling towers using steady state models with time invariant parameters, this is a significant find as final values can be out by a factor of $1.8 \%$. If looking at operation over the course of the year, $1.8 \%$ could be the difference between thousands of dollars. The average operating cost of case 6 yields significant savings over Base Case B. In terms of the average operating cost, case 6 represents a saving of $29.896 \%$ over Base Case B. Comparing case 6 to case 4, the difference between the average costs over the 24 hours amounted to be $5 \mathrm{E}-06 \%$, which is negligible.

As it is the makeup water, which primarily dictates the height of the water level, the results across all three optimisation schemes are in good agreement with expectations as shown in Figure 20. For cases 4 and 6, as the makeup water is minimised to a fixed rate between 00:00 and 23:00, it reduces the cyclic loading on the pump compared to running the pump with a variable speed over a 24-hour period. By having the pump flowrate fixed until 23:00 and turning the pump off after 23:00, this approach drastically improves the life of the pump. As the seawater contains lots of scalants and other dissolved solids, the one or two-hours where it is turned off, this time period can be used for maintenance work. Furthermore, the average makeup flowrate was found to be very close between cases 4 and 6 . This indicates that both of the results of the case studies were next to identical, although the objective function for each one was different. Results of case 5 had shown that there is a $38.8 \%$ reduction compared to Base Case B of the makeup water flowrate and had remained constant over the 24-hour period.

These results obtained for seawater are in stark contrast to those results obtained for freshwater. This is down to the fact that there is a constraint on the number of cycles, which had an upper limit of two to minimise fouling and solid deposition within the cooling tower fill and cooler network. As there is such a low value of the number of cycles, the scope of optimisation is significantly reduced.

Table 6 shows the pumping power required by the blowdown and makeup water streams. These results show that it is case 6 , which yields the biggest savings over Base Case B. With respect to the blowdown stream, the pumping power required for cases 5 and 6 were found to be almost the same. However, in terms of the power required for the makeup water stream, it is seen that case 6 yields the largest savings. This is in line with expectations as it was the makeup water stream which was to be minimised. 


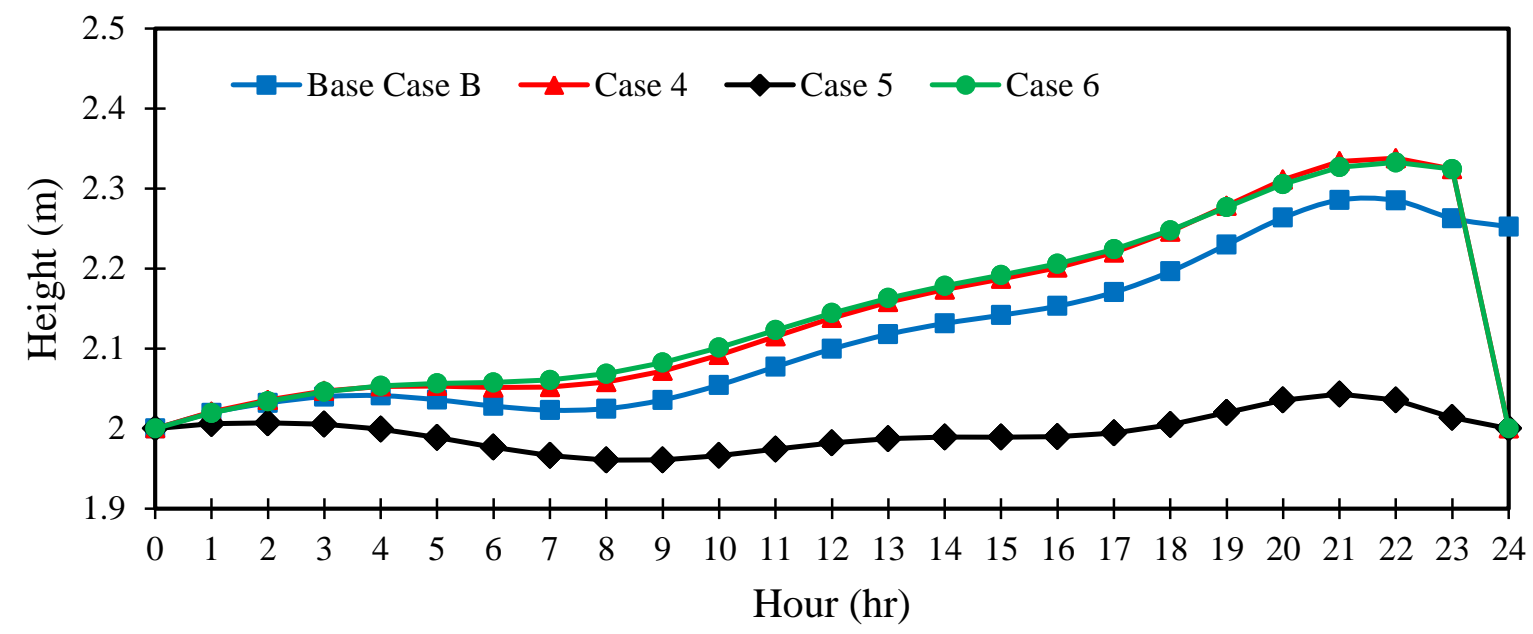

Figure 18. Seawater medium optimisation - height of collection basin

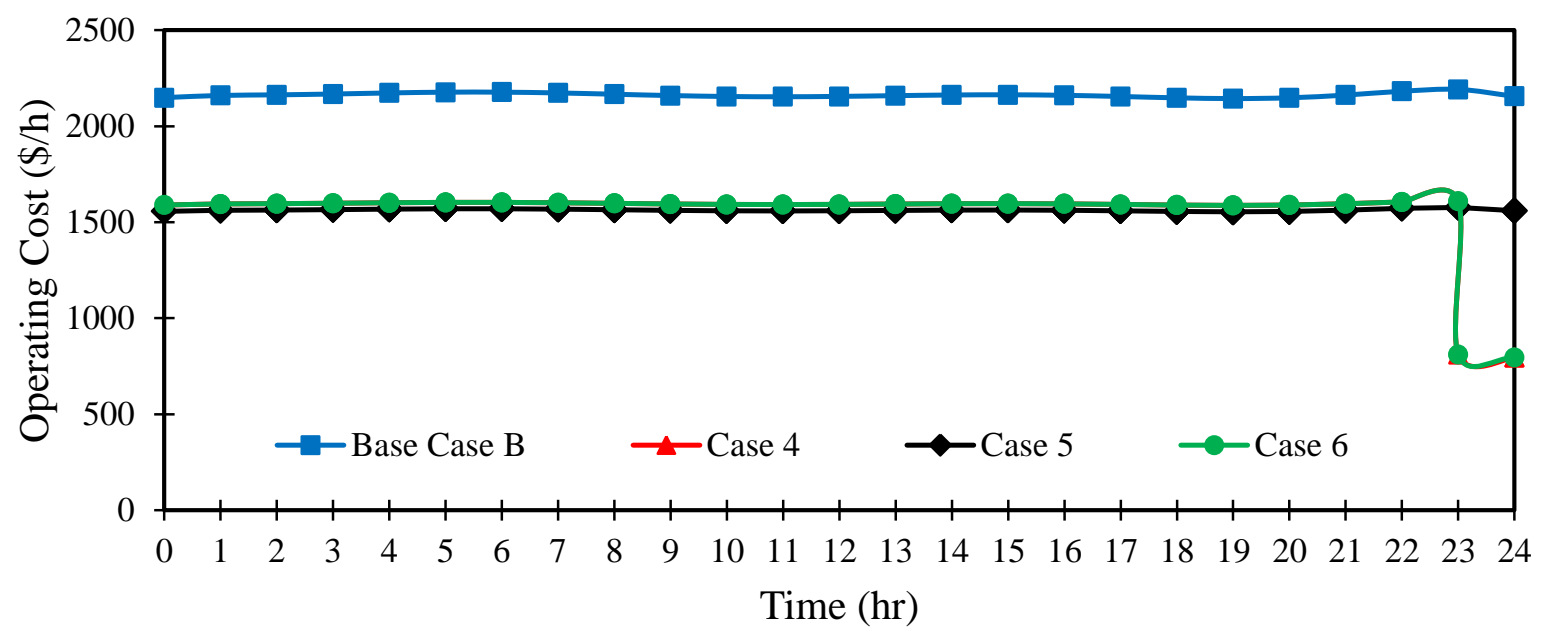

Figure 19. Seawater medium optimisation - operating cost

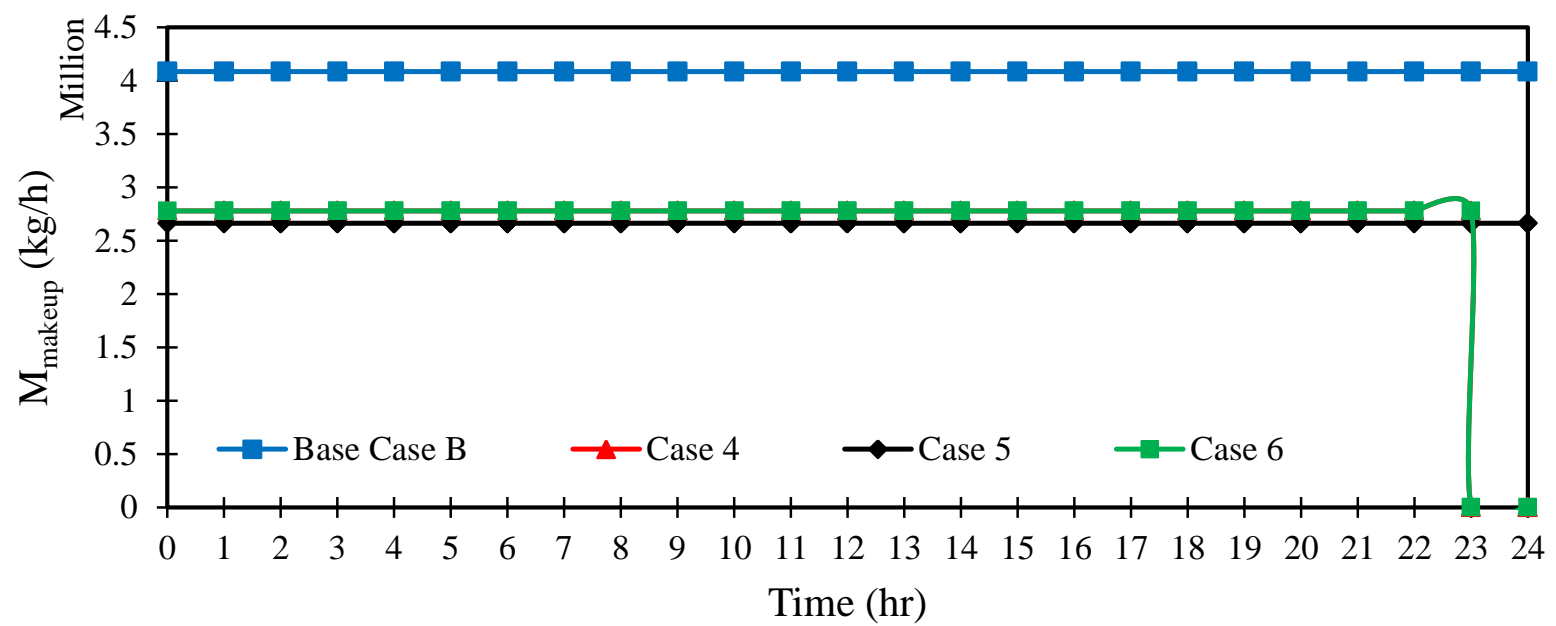

Figure 20. Seawater medium optimisation - mass flow of makeup water 
Table 6: Optimised average pump requirements for cases 4, 5 and 6

\begin{tabular}{ccc|cc|cc} 
& \multicolumn{6}{c}{ Average Pumping Power (kW) } \\
\cline { 2 - 7 } & \multicolumn{2}{c}{ Case 4 } & \multicolumn{2}{c}{ Case 4 } & \multicolumn{2}{c}{ Case 6 } \\
\cline { 2 - 7 } & Blowdown & Makeup & Blowdown & Makeup & Blowdown & Makeup \\
\hline Base Case B & 31.78 & 48.84 & 31.78 & 48.84 & 31.78 & 48.84 \\
\hline Optimised & 23.42 & 38.71 & 15.90 & 31.841 & 15.90 & 31.19 \\
\hline \% Change & -26.30 & -20.74 & -49.96 & -34.81 & -49.96 & -36.14 \\
\hline
\end{tabular}

\subsection{Optimisation Summary}

Table 7 shows the average operating costs amongst all 6 different case studies undertaken in this paper. It can be seen that the fresh water is significantly cheaper compared to the seawater due to the fact that it has a higher cycles of concentration count which reduces the amount of water that is used for blowdown. However, it can be said that the results of this optimisation study have saved significant amounts of money compared to their respective Base Cases A and B. As each different objective function induces a different system response, the selection of the objective function rests upon the requirements of the plant operator. Upon analysis of the total pumping cost, it can be seen that savings were also found. However, the cost of pumping was insignificant in comparison to the chemical treatment and makeup water costs.

Table 7. Average operating costs when utilising different objective functions

\begin{tabular}{c|c|c|c|c|c|c|c|c}
\hline Medium & \multicolumn{4}{|c|}{ Fresh water } & \multicolumn{3}{c}{ Seawater } \\
\hline Case & $\begin{array}{c}\text { Base } \\
\text { Case A }\end{array}$ & $\mathrm{C} 1$ & $\mathrm{C} 2$ & $\mathrm{C} 3$ & $\begin{array}{c}\text { Base } \\
\text { Case B }\end{array}$ & $\mathrm{C} 4$ & $\mathrm{C} 5$ & C6 \\
\hline $\begin{array}{c}\text { Objective } \\
\text { Function }\end{array}$ & - & $\begin{array}{c}\text { Operating } \\
\text { Cost }\end{array}$ & $\begin{array}{c}\mathrm{M} \\
\text { (Holdup) }\end{array}$ & $\mathrm{m}_{\text {makeup }}$ & - & $\begin{array}{c}\text { Operating } \\
\text { Cost }\end{array}$ & $\begin{array}{c}\mathrm{M} \\
\text { (Holdup) }\end{array}$ & $\mathrm{m}_{\text {makeup }}$ \\
\hline $\begin{array}{c}\text { Operating } \\
\text { Cost } \\
(\$ / \mathrm{hr})\end{array}$ & 1828.08 & 1147.36 & 1234.51 & 1140.57 & $\$ 2155.41$ & 1562.95 & 1756.07 & 1562.95 \\
\hline $\begin{array}{c}\text { Pumping } \\
\text { Cost } \\
(\$ / \mathrm{hr})\end{array}$ & 0.24 & 0.23 & 0.23 & 0.23 & 0.25 & 0.24 & 0.24 & 0.24 \\
\hline $\begin{array}{c}\text { Chemical } \\
\text { Treatment } \\
\text { Cost } \\
(\$ / \mathrm{hr})\end{array}$ & 1157.24 & 597.98 & 597.98 & 597.98 & 1157.24 & 976.99 & 754.46 & 787.26 \\
\hline $\begin{array}{c}\text { Makeup } \\
\text { Water } \\
\text { Cost } \\
(\$ / \mathrm{hr})\end{array}$ & 598.14 & 488.27 & 444.98 & 489.53 & 598.11 & 597.98 & 597.99 & 597.99 \\
\hline
\end{tabular}




\section{Conclusions}

In this work, a dynamic model was developed for natural draft cooling towers subjected to variable air temperature and humidity ratio. Transient results were found for the evaporation rate and the amount of water leaving the fill after cooling. As there was transient behaviour within the cooling tower, the next evolutionary step was taken where the collection basin at the bottom was modelled. Equations were developed from first principles for the mass and energy balances. Two base case simulations (A and B) were undertaken where one cooling medium was freshwater and the other was seawater. Large discrepancies were found between the two as the freshwater simulation had a much higher rate of accumulation due to a higher number of cycles of concentration. Both scenarios were optimised with respect to three objective functions; the operating cost, the accumulation within the collection basin and the makeup water flowrate. It was found that on average the fresh water system had a much lower operating cost than that of seawater across all three objective functions.

\section{Nomenclature}

$A_{z}$ : Interfacial area $\left(\mathrm{m}^{2}\right)$

$C_{p}:$ Specific heat capacity $(\mathrm{J} / \mathrm{kg})$

$g:$ Gravity $\left(\mathrm{m} / \mathrm{s}^{2}\right)$

$L e_{f}$ : Lewis factor

$m$ : Mass flowrate $(\mathrm{kg} / \mathrm{s})$ or $(\mathrm{kg} / \mathrm{hr})$

M: Mass accumulation $(\mathrm{kg})$

$M e:$ Merkel number from Poppe Analysis

$n_{\text {cycle }}$ : Number of cycles of concentration

$O C$ : Operating cost $(\$ / \mathrm{hr})$

$P P$ : Pumping power $(\mathrm{kW})$

$r_{o}$ : Latent heat of vaporisation $(\mathrm{J} / \mathrm{kg})$

$t$ : Time (sec or hour)

$T$ : Temperature $\left({ }^{\circ} \mathrm{C}\right)$ or $(\mathrm{K})$

$T_{a}$ : Temperature of air $\left({ }^{\circ} \mathrm{C}\right)$ or $(\mathrm{K})$

$V$ : Volume $\left(\mathrm{m}^{3}\right)$

$X$ : Humidity ratio $(\mathrm{kg} / \mathrm{kg})$

$X_{S}$ : Saturation humidity ratio $(\mathrm{kg} / \mathrm{kg})$ 
$\alpha$ : Heat transfer coefficient

$\beta$ : Mass transfer coefficient

$\eta$ : Pump efficiency

$\rho$ : Density $\left(\mathrm{kg} / \mathrm{m}^{3}\right)$

Subscripts

$a$ : Air

$c b$ : Collection basin

$e$ : Evaporation

$H E N$ : Heat exchanger network

$i$ : Inlet

m: Makeup

$o$ : Outlet

$w$ : Water

$w b$ : Wet bulb

\section{References}

AccuWeather., 2017. Johannesburg Hourly Weather - AccuWeather Forecast for Gauteng South Africa. [online] Available at: http://www.accuweather.com/en/za/johannesburg/305448/hourly-weatherforecast/305448?hour=33 [Accessed 24 Mar. 2017].

Al-Nimr M., 1998. Dynamic thermal behaviour of cooling towers. Energy Conversion and Management, 39(7), 631-636.

Alsadaie S. and Mujtaba I., 2017. Dynamic modelling of Heat Exchanger fouling in multistage flash (MSF) desalination. Desalination, 409, pp.47-65.

Buecker B., 1997. Power plant water chemistry. 1st ed. Tulsa, OK: PennWell Pub., 162.

Cortinovis G. Paiva J., Song T. and Pinto J., 2009. A systemic approach for optimal cooling tower operation. Energy Conversion and Management, 50(9), 2200-2209.

Dhorat A., Al-Obaidi M. and Mujtaba I., 2018. Numerical Modelling and Sensitivity Analysis of Natural Draft Cooling Towers. Chemical Product and Process Modeling.

Gololo K. and Majozi T., 2011. On Synthesis and Optimization of Cooling Water Systems with Multiple Cooling Towers. Industrial \& Engineering Chemistry Research, 50(7), 3775-3787. 
He S., Gurgenci H., Guan Z., Hooman K., Zou Z. and Sun F., 2016. Comparative study on the performance of natural draft dry, pre-cooled and wet cooling towers. Applied Thermal Engineering. 99,pp.103-113.

Hensley J., 1985. Cooling tower fundamentals. 1st ed. Mission, Kan.: Marley Cooling Tower Co., 37.

Klimanek A., 2013. Numerical Modelling of Natural Draft Wet-Cooling Towers. Archives of Computational Methods in Engineering, 20(1), 61-109.

Kloppers J. and Kröger D., 2004. Cost optimization of cooling tower geometry. Engineering Optimization, 36(5), 575-584.

Kloppers J. and Kröger D., 2005. The Lewis factor and its influence on the performance prediction of wet-cooling towers. International Journal of Thermal Sciences. 44(9), 879-884.

Ortega J., Gonzalez M. and Gutierrez A., 2010. Optimization model for re-circulating cooling water systems. Computers and Chemical Engineering, 34(2), 177-195.

Panjeshahi M., Ataei A., Gharaie M. and Parand R., 2009. Optimum design of cooling water systems for energy and water conservation. Chemical Engineering Research and Design, 87(2), 200-209.

PSE., 2004. gPROMS Advanced User Guide. London: Process Systems Enterprise Limited, 20.

Williamson N., Behnia M. and Armfield S., 2008. Thermal optimization of a natural draft wet cooling tower. International Journal of Energy Research, 32(14), 1349-1361. 